\title{
RECENT DYNAMIC MEASUREMENTS AND CONSIDERATIONS FOR AERODYNAMIC MODELING OF FIGHTER AIRPLANE CONFIGURATIONS
}

\author{
Jay M. Brandon* and John V. Foster* \\ NASA Langley Research Center \\ Hampton, VA 23681-0001
}

\begin{abstract}
As airplane designs have trended toward the expansion of flight envelopes into the high angle of attack and high angular rate regimes, concerns regarding modeling the complex unsteady aerodynamics for simulation have arisen. Most current modeling methods still rely on traditional body axis damping coefficients that are measured using techniques which were intended for relatively benign flight conditions. This paper presents recent wind tunnel results obtained during largeamplitude pitch, roll and yaw testing of several fighter airplane configurations. A review of the similitude requirements for applying sub-scale test results to fullscale conditions is presented. Data is then shown to be a strong function of Strouhal number - both the traditional damping terms, but also the associated static stability terms. Additionally, large effects of sideslip are seen in the damping parameter that should be included in simulation math models. Finally, an example of the inclusion of frequency effects on the data in a simulation is shown.

\section{Nomenclature}

b wing span

c mean aerodynamic chord

$\mathrm{C}_{1} \quad$ rolling moment coefficient

$\mathrm{C}_{1} \quad$ static lateral stability derivative

$\mathrm{C}_{\mathrm{p}}$ body axis roll damping derivative

$\mathrm{C}_{1_{\mathrm{r}}} \quad$ rolling moment due to yaw rate derivative

$\mathrm{C}_{\Omega} \quad$ stability axis roll damping derivative
\end{abstract}

\footnotetext{
*Aerospace Engineer, Senior Member AIAA
}

Copyright (C) 1998 by the American Institute of Aeronautics and Astronautics, Inc. No copyright is asserted in the United States under Title 17, U.S. Code. The Government has a royalty-free license to exercise all rights under the copyright claimed herein for Government purposes. All other rights are reserved by the copyright owner.

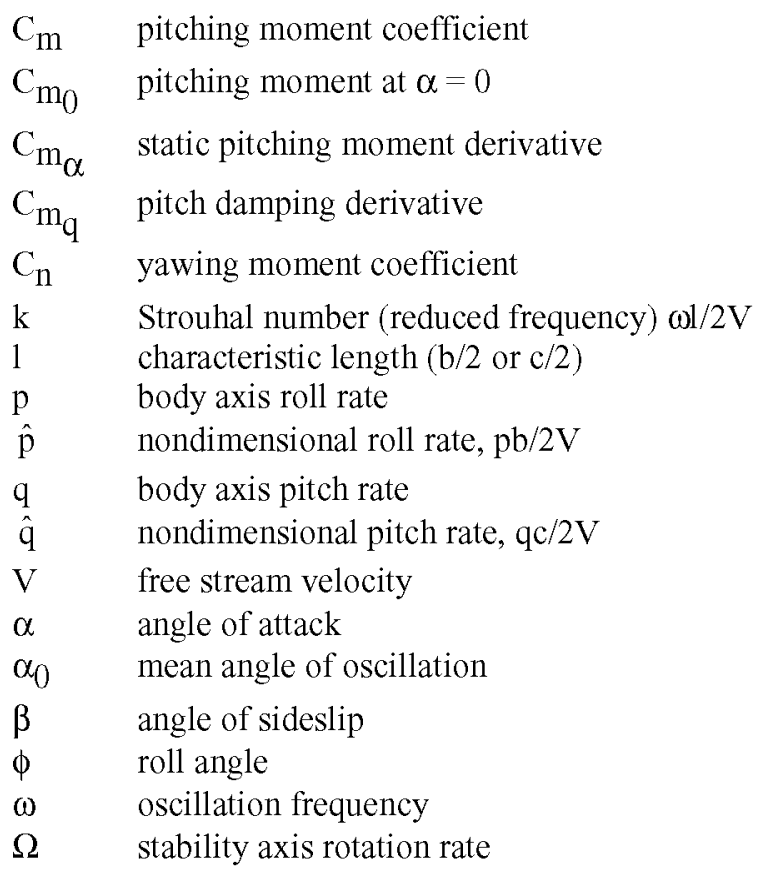

\section{Introduction}

At high angles of attack, unsteady aerodynamic effects may have a major impact on the maneuverability and controllability of an airplane. Currently, some modern fighter airplanes are capable of performing transient maneuvers involving high pitch rates to extreme angles of attack; this has been graphically demonstrated in the so-called "Cobra maneuver" flown by Soviet aircraft at recent airshows. The advent of innovative high- $\alpha$ control effectors such as thrust vectoring and forebody controls will enable even greater capability to effectively exploit a substantially enlarged envelope for air combat. The impact of unsteady aerodynamic effects on airplane flight dynamics (including stability and control effects during rapid high angle-of-attack maneuvers) and a practical means of utilizing these unsteady effects need to be addressed.

Because current generation developmental aircraft programs are relying more heavily on simulation based predictions of airplane dynamics to reduce risk and cost of flight test programs, accurate prediction of aircraft 
dynamics is critical. With airplanes operating in the highly nonlinear flow regimes, including post stall angles of attack, with substantial angular rates, prediction of departures and flight dynamics have received increased attention. Several efforts have been undertaken to address this area, including guidelines development for preliminary design ${ }^{1}$, improved testing techniques ${ }^{2}$, and improved analysis techniques such as in the prediction of falling leaf motions $s^{3}$. Nevertheless, no industry standard or accepted procedures exist which provide for high confidence prediction of airplane dynamics in the high angle of attack, and high angular rate environment. Some efforts have been made which seem to improve the simulation predictive capabilities ${ }^{4}$, however these techniques still do not always provide good simulation predictions. Most simulation development programs today rely on static wind tunnel measurements, rotary balance measurements and forced oscillation measurements to develop the aerodynamic model.

A significant amount of research has been conducted in the area of unsteady aerodynamics at high angles of attack. Results for two-dimensional airfoils show substantial force overshoots for pitching and plunging airfoils $^{5-7}$. Wind tunnel experiments have been conducted using delta wings undergoing pitching and plunging oscillations to determine vortical flow characteristics during large-amplitude motions ${ }^{8-10}$. The primary focus of the studies was the dynamics of the leading-edge vortex while the wing was undergoing motion. Under these conditions, the vortex burst point location was observed to lag the static location during pitch-up and pitch-down motions. Water tunnel results ${ }^{11}$ have shown vortex lag times of up to 30 convective time units (time required for an air particle to travel across the wing), which is a much slower response than that normally associated with twodimensional dynamic stall phenomena. Research was conducted on a series of wings of different sweep angles and showed that the magnitude of the unsteady aerodynamic effects decreased as the sweep angle increased $^{12}$. Additional tests of semi-span models with various wing sweeps were reported in reference 13. Reference 14 extended this work to a representative three-dimensional configuration, tested at zero sideslip and with no control deflections, which showed significant force and moment increments due to dynamic stall effects. Measured persistence times of the dynamic effects were also presented. Reference 15 presented detailed wing surface pressure measurements of a straked-wing fighter model undergoing pitch oscillations. Force and moment measurements from water tunnel studies with a series of straked-wing models have also been reported ${ }^{16}$. Recent studies have been reevaluating data reduction techniques from conventional wind tunnel tests ${ }^{17}$, and mathematical structures for simulation modeling ${ }^{18-23}$.

This paper presents results from tests of an F-16XL model in the NASA Langley 12 Foot Low Speed Wind Tunnel undergoing pitching motions, and another F$16 \mathrm{XL}$ along with a modern fighter model in the Langley 14 -by -22 Foot Subsonic Wind Tunnel undergoing roll and yaw oscillations at various angular rates and amplitudes. These results can be used to help develop better understanding of the complex dynamic aerodynamics and in turn aid in the development of better mathematical modeling techniques.

\section{Models and Test Apparatus}

Pitch axis static and dynamic tests were conducted in the NASA Langley 12-Foot Low-Speed Wind Tunnel using a 0.10 -scale F-16XL model (figure 1). The model was mounted on a dynamic test rig through a sixcomponent strain-gauge balance. The dynamic test rig is a computer controlled, hydraulically actuated system that was sting-mounted on a C-strut support system. The mounting arrangement rotated the model about the reference center of gravity location of $0.558 \mathrm{c}$, over an angle of attack range of $-5^{\circ}$ to $80^{\circ}$. The maximum capability of the dynamic test rig was $260 \mathrm{deg} / \mathrm{sec}$ pitch rate, and $2290 \mathrm{deg} / \mathrm{sec}^{2}$ pitch acceleration. Further description of the dynamic test rig may be found in reference 2 . The tests were conducted at a dynamic pressure of 4 psf resulting in a Reynolds number of $10^{6}$ based on the mean aerodynamic chord.

All data were obtained with the leading-edge flaps at $0^{\circ}$ deflection. Static data were obtained for angles of attack from $-5^{\circ}$ to $80^{\circ}$ at zero sideslip and zero deflection of trailing-edge surfaces (flaperons and elevons). Additional data were obtained for four values of sideslip, $\left(\boldsymbol{\beta}=-5^{\circ},-10^{\circ},-20^{\circ}\right.$, and $\left.-30^{\circ}\right)$, and for two values of symmetric trailing-edge control surface deflections $\left(\delta_{\mathrm{e}}=-20^{\circ}\right.$ and $\left.20^{\circ}\right)$. Oscillatory data were obtained at initial angles of attack $\left(\alpha_{0}\right)$ between $20^{\circ}$ and $60^{\circ}$ at several frequencies and amplitudes. The effects of sideslip and control surface deflections were measured only at an initial angle of attack of $35^{\circ}$ and two frequencies $(0.6$ and $1.41 \mathrm{~Hz})$ at the same sideslip values and control deflections as in the static test. Ramp tests (constant pitch rate motions) were conducted similarly with various positive and negative pitch rates, and various starting and ending angles of attack.

Data recorded during the test runs included a linear variable differential transformer reading from the dynamic test rig to determine the pitch angle, six- 
component force and moment data from a strain-gauge balance, and wind tunnel dynamic pressure. Data were sampled at $100 \mathrm{~Hz}$ with an in-line $100 \mathrm{~Hz}$ low pass filter. All data channels were subsequently filtered using a $6 \mathrm{~Hz}$ low pass filter. The combination of model pitch rates and tunnel speeds allowed for a realistic representation of full-scale maneuvering conditions within the capabilities of current and projected future high performance airplanes.

Roll and yaw axis forced oscillation data were obtained with an 0.18-scale F-16XL model and other fighter models using a forced oscillation rig in the 14 -by -22 Foot Subsonic Wind Tunnel (figure 2). Data were obtained for 4 amplitudes of oscillation and a range of frequencies to cover a broad range of angular rates and reduced frequency combinations. Data were sampled at $200 \mathrm{~Hz}$ with an inline $100 \mathrm{~Hz}$ anti-aliasing filter and further filtered during post processing with a low pass filter similar to the pitch data discussed above. Ensemble averaging was conducted over approximately 40 cycles of the data before using it to develop regression models. Time history format of the data were obtained and analyzed in addition to in-phase and out-of-phase coefficient data used in traditional forced oscillation testing.

\section{Similitude Considerations}

Full-scale predictions using sub-scale models rely on satisfying a set of similitude requirements. A summary of some of these requirements is shown in figure 3 . As can be seen from the figure, accurate predictions of fullscale characteristics require correlation of many, sometimes conflicting, similitude parameters. When predicting static characteristics, for example, flow angles and control surface deflections need to be identical between sub-scale and flight. Mach number and Reynolds numbers also need to be maintained constant between model and flight for the data to be valid as tested. When the airplane is undergoing rotational motions, reduced angular velocity and Strouhal number (frequency content) need to match between model and flight according to the nondimensional analysis results. Finally, for unconstrained model testing, in order to achieve the same (scaleable) flight dynamics, the model needs to be dynamically scaled as shown.

Various types of wind tunnels and test techniques have been developed largely to address the many similitude requirements. Traditional wind tunnel testing has tried to take into account all of the static model considerations, and also the angular velocity scaling. The frequency-dependence in the data denoted by the Strouhal number ( $k$, or reduced frequency) was documented in the 1950's, however this effect typically is not addressed in current testing or simulation modeling. The only test method currently in use to obtain $\mathrm{k}$ effects is the forced oscillation test, however this data typically is modeled as a linear derivative in non-dimensional rate for only one $\mathrm{k}$ value which is usually picked based on estimates of Dutch-roll frequency or short period frequency. Although this method worked well for airplanes in the past, the new realities of operating in very non-linear flow regimes may have invalidated some of the assumptions and simplifications used previously.

A rigorous modeling approach might look something like:

$$
C_{i}=f(\alpha, \beta, \delta, M, \operatorname{Re}, \Omega l / V, k)
$$

where each coefficient is dependent on all of the nondimensional parameters. The current modeling approach looks more like:

$$
\begin{array}{rlrl}
\mathrm{C}_{\mathrm{i}}= & \mathrm{f}(\alpha, \beta, \delta) & & \text { (measured static data) } \\
& +\mathrm{f}(\mathrm{M}) & & \text { (Mach number effects) } \\
& +\mathrm{f}(\mathrm{Re}) & & \text { (Reynolds number corrections) } \\
& +\mathrm{f}(\Omega 1 / \mathrm{V}) & & \text { (rotary balance data) } \\
& +\mathrm{f}(\mathrm{pl} / \mathrm{V}, \mathrm{ql} / \mathrm{V}, \mathrm{rl} / \mathrm{V}) \text { (forced oscillation data) }
\end{array}
$$

where effects are assumed independent, and also that the linear superposition principles apply. This approach, although in standard use, is based on broad assumptions, which at least until recently, appear to have been justified.

\section{Pitch Axis Results}

Several approaches have recently been applied to improve modeling the observed nonlinear, unsteady aerodynamic responses seen during large amplitude pitching motions. To date, there is no consensus of approaches to model observed effects. Little validation of proposed modeling methods is available, and the literature is dominated by theoretical and mathematical studies. Figure 4 shows some basic characteristics observed in oscillatory motions. The data show the effect of oscillation amplitude on lift and pitching moment for constant maximum pitch rates. As can be seen, large variation of aerodynamic parameters is apparent due to the amplitude. At $\alpha=36^{\circ}$, (midpoint of oscillation cycle), all normal state variables $(\alpha, \beta, q)$ are identical; however, the aerodynamic coefficients are very different.

Results from constant pitch rate motions are shown in figure 5. These data were all obtained from ramp 
motions beginning at $\alpha=0^{\circ}$ (positive pitch rates) or $\alpha=75^{\circ}$ (negative pitch rates). The coefficient increments were obtained by subtracting the quasi-static results $(\mathrm{qc} / 2 \mathrm{~V}=0.0004)$ from the dynamic results. These increments show large variations in lift and pitching moment which are nonlinear functions of $\alpha$ and non-dimensional rate. The largest increments seen during the pitch-up motions were at the highest angles of attack. The largest increments seen (especially in lift) during the pitch-down maneuvers were for the moderate angle of attack range near the steady stall angle of attack. This phenomenon is consistent with flow lag effects seen and reported in numerous previous studies. At positive pitch rates the flowfield formation dynamically lags the static conditions for a given angle of attack and results in a delay of the separated flow fields. This gives rise to the large increments in lift at the high angles of attack and pitch rate. At negative pitch rates, again the flow formation lags, such that the re-establishment of vortex systems and attached flow occurs at lower angles of attack than would be the case for static conditions.

The effect of oscillation amplitude is shown in figure 6. The data show that there are large effects of amplitude at the negative pitch rates, which increase with amplitude. Positive pitch rate results are relatively minor with amplitude changes. This illustrates the importance of the "time history effect" which is why modeling dynamic data without either time dependence or new state variables may lead to erroneous results. At any given vertical crosscut on figure 6 , the standard state variables ( $\alpha, \mathrm{q}, \dot{\alpha})$ are equivalent; however, large discrepancies in the data exist. The difference is that for each successive increase in amplitude, the model previously was further into vortex-dominated flow or separated flow regimes before arriving at the given angle of attack. The flow lags mentioned previously then contribute to the differences seen in the data. For positive pitch rates through $\alpha=21^{\circ}$, little change is seen with amplitude. This is because the model is arriving at the $\alpha=21^{\circ}$ point from lower, primarily attached flow conditions. The time scales associated with flow lags during attached flow are very fast, and do not result in significant increments. Figure 7 shows similar data at $\alpha=51^{\circ}$. At this angle of attack, the large increments are observed for the positive pitch rates. Again, similar to the previous example, the small amplitude shows the smallest increments. At $\alpha=51^{\circ}$, and an oscillation of $\pm 5 \mathrm{deg}$, the model remains in separated flow with the leading-edge vortex system burst. Oscillations inside a completely separated flow regime without leading-edge vortex effects result in very little dynamic increments. When the amplitude increases, and vortex formation occurs during the oscillation cycle, then the dynamic effects become significant and large increments are seen. This behavior is very similar to that discussed in previous reports regarding crossing of bifurcation points.

The experimental study did not conduct sufficient testing to make extensive comparisons of amplitude and rate effects with constant Strouhal numbers (for similitude requirements), but linear derivative estimates could be calculated for pitch damping and then compared with ramp data for trends. A postulated mathematical model;

$$
\mathrm{C}_{\mathrm{m}}(\alpha, \mathrm{k})=\mathrm{C}_{\mathrm{m}_{0}}(\mathrm{k})+\mathrm{C}_{\mathrm{m}_{\alpha}}(\mathrm{k})^{*} \alpha+\mathrm{C}_{\mathrm{m}_{\mathrm{q}}}(\mathrm{k})^{*} \mathrm{qc} / 2 \mathrm{~V}
$$

was used and considered to be a function of $\mathrm{k}$. Parameter estimation using the method of reference 24 was conducted using this model. The fit of the data, for constant $\mathrm{k}$ values was very good and generally accounted for over 98 percent of the variation seen in the data. Figure 8 shows a comparison of the dynamic increments, computed as previously discussed, for constant rate ramp data with the results obtained from the regression model for the $\mathrm{C}_{\mathrm{m}_{\mathrm{q}}}(\mathrm{k})$ term at $\alpha=36^{\circ}$. The data show a large dependence on $\mathrm{k}$. At large values of $\mathrm{k}$, stable damping characteristics are seen. As $\mathrm{k}$ decreases, the pitch damping stability decreases and becomes unstable at the low values of $\mathrm{k}$. The trend appears to be consistent with the ramp results which show unstable damping at this angle of attack.

Limited testing at sideslip was also conducted during the large amplitude pitching tests. Figure 9 shows the general character of results in sideslip. The lags in the vortex burst and flow field formation/reattachment result in the hysteresis loops seen in the data. These lags are seen to significantly delay the unstable characteristics both in roll and yaw to higher angles of attack than for static conditions. The effect of the vertical tail on the dynamic increments in sideslip is shown in figure 10. These data show that the vertical tail had very little impact on rolling moment increments. The unsteady effects did impact the flow around the tail giving directional stability improvements dynamically compared to the static conditions at $\alpha=36^{\circ}$.

\section{Roll and Yaw Axes Results}

Roll and yaw forced oscillation tests were conducted in the NASA Langley 14 -by -22 Foot Subsonic Tunnel using the standard low-speed forced oscillation test rig previously used in the NASA Langley 30 -by -60 Foot Tunnel. Figure 11 shows a typical time history of rolling moment and roll angle of the F-16XL model 
oscillation at $\alpha=34^{\circ}$ at an amplitude of $30^{\circ}$. Figure 12 shows rolling moment versus roll angle for 40 oscillation cycles illustrating the repeatability of the dynamic data. Several methods have been proposed and used in analysis of forced oscillation data. An integrated technique ${ }^{25}$ which involves using in-phase and out-of-phase components to determine damping coefficients is the predominately used technique. Another technique, sometimes referred to as the "single point method", utilizes only the points through the midpoint of the oscillation cycle, where $\alpha$ and $\beta$ are the known nominal values, angular rate is a maximum (absolute) value, and angular acceleration is zero. A comparison of the two methods was made and is shown in figure 13. The single-point values were obtained by subtracting the rolling moment coefficient at negative rate from the positive rate value, and dividing by the sum of the absolute values of the rates. The data were obtained at three amplitudes, and the frequency of the oscillation was varied to maintain a constant nondimensional roll rate through the midpoint of the oscillation cycle of $\hat{p}=0.04$. The results show a large effect of amplitude, but both data reduction methods resulted in the same values of roll damping.

Rolling moment data for the F-16XL model at various angles of attack and a constant $\hat{p}$ through the midpoint of the oscillation cycle of $\hat{p}=0.04$ is shown in figure 14. With constant $\hat{\mathrm{p}}_{\text {max }}$ values, the points at the midpoint of the oscillation cycle have identical state values of $\alpha, \beta$, and $p$, and all have zero roll acceleration. For angles of attack below $20^{\circ}$, the effect of the amplitude of the oscillation is very small, evidenced by the coincidence of all of the data at $\phi=$ $0^{\circ}$. The positive values of $\mathrm{C}_{1}$ at $\phi=0^{\circ}$ correspond to negative roll rate, and correspondingly, the negative values of $\mathrm{C}_{1}$ at $\phi=0^{\circ}$ is for positive roll rate. The rolling moment increment at $\phi=0^{\circ}$ between the upper and lower segments of each curve is an indication of the traditional rate damping. Because $\phi$ and $\beta$ are inversely correlated, the slope of the curve is an indication of the dihedral effect.

For angles of attack between $25^{\circ}$ and $40^{\circ}$, large variation of the data at $\phi=0^{\circ}$ are seen between the oscillations at different amplitudes. This effect is analogous to that described previously in pitching motions as "time history" effects. Finally, as $\alpha$ is increased further, the effect of amplitude is decreased and becomes unimportant for this configuration.

These effects may be very configuration dependent. Depending on dominant flow structure topology during the motions, time history effects could be quite different. Several other configurations were tested similarly to the F-16XL to investigate this possibility. Rolling moment data for a modern fighter (fig. 2) with low-swept wings, a highly swept LEX, and a long forebody are shown in figure 15 . This configuration shows much less sensitivity to roll oscillation amplitude than the F-16XL configuration. Additionally, the trend of the data with amplitude is opposite that of the F16XL. Finally, the effects of amplitude persist to the extreme $\alpha$ 's instead of decreasing, as was the case for the F-16XL. Flow visualization or unsteady pressure measurements would be needed to explain the physics behind the differences.

In order to identify key parametric effects, make comparisons to current data models, and allow comparisons to other techniques, a simple model was initially chosen to represent the forced oscillation data. The same regression technique discussed previously in the pitch results section was used to identify a model of the form:

$$
\mathrm{C}_{\mathrm{l}}(\alpha, \beta, \mathrm{k})=\mathrm{C}_{\mathrm{p}}(\alpha, \beta, \mathrm{k}) * \mathrm{pb} / 2 \mathrm{~V}+\mathrm{C}_{\mathrm{l}}(\alpha, \beta, \mathrm{k}) * \beta
$$

This model allowed correlation of parameters with $\mathrm{k}$, and allowed for the calculation of a parameter to compare with conventional rotary balance data. Rotary balance data is obtained by rotating the model at various angles of attack and sideslip about the wind velocity vector.

The regression model resulted in very good fits of the data below $\alpha=35^{\circ}$ and above $\alpha=45^{\circ}$. At the intermediate angles of attack, the fits were still qualitatively good, but higher order terms would have to be included in the model to follow the nonlinear shape of the data. An example of the regression model results is shown in figure 16 for the F-16XL at $\alpha=30^{\circ}$ and oscillation amplitudes of $5^{\circ}-30^{\circ}$. The data show that the damping parameter rapidly decreases as $\mathrm{k}$ increases, while the "static" term $\left(\mathrm{C}_{1}\right)$ increases with increasing $\mathrm{k}$ values. Also plotted is the static wind tunnel value obtained for $\mathrm{C}_{\beta}$ reported in a previous test $^{26}$. The trend of $\mathrm{C}_{\beta}$ obtained dynamically appears to correlate well with the $\mathrm{C}_{\beta}$ obtained previously in a static test.

Figure 17 shows the regression results for roll oscillations at $\alpha=40^{\circ}$. As previously mentioned, this angle of attack range produced poorer fits to the wind tunnel data, and consequently, more data scatter is 
observed. Least-squares curve fits to the regression model results are shown and indicate the same trends seen at $\alpha=30^{\circ}$. Again, the static data previously obtained is plotted at $\mathrm{k}=0$ for reference and the dynamically derived values appear to have good agreement. Unfortunately, amplitude effects are still present, particularly in the mid-angle-of-attack range as discussed above. Figure 18 shows the effect of amplitude on rolling moment coefficients at $\alpha=40^{\circ}$. Amplitude effects are present for yaw oscillation data at the higher amplitudes, shown in figure 19.

Figure 20 shows a summary of the effects of $k$ on rolling moment derivatives over a range of angles of attack. The data show that at low angles of attack, the effects of $\mathrm{k}$ are small. At the moderate angles of attack, $k$ effects are very large for both $\mathrm{C}_{1}$ and $\mathrm{C}_{1}$. As angle of attack is further increased, the effect of $\mathrm{k}$ decreases again. This character of the data indicates that a reasonable model may be achievable by including the $\mathrm{k}$ effects over a fairly narrow range of angle of attack.

Combining the rolling moments due to roll rate, and yaw rate with the formula

$$
\mathrm{C}_{\Omega}=\mathrm{C}_{\mathrm{p}} \cos (\alpha)-\mathrm{C}_{\mathrm{r}_{\mathrm{r}}} \sin (\alpha)
$$

enables a comparison with rotary balance data which is obtained by steady rotation $(\mathrm{k}=0)$ about the wind velocity vector. Since $\mathrm{C}_{\mathrm{I}_{\Omega}}$ is a steady value, and the $\mathrm{C}_{\mathrm{p}}$ and $\mathrm{C}_{\mathrm{r}}$ values are unsteady measurements (functions of $\mathrm{k}$ ), vectorally combining these values, as is currently done in some simulation models using the Kalviste $^{4}$ or other techniques may be misleading if large dependencies on $\mathrm{k}$ exist for the body axis parameters. A comparison of forced oscillation and rotary data is shown in figure 21 . The rotary data is plotted at $\mathrm{k}=0$, and is shown as two boxes on the plot, which covers the range slopes of the nonlinear data.

The intent of inclusion of the Strouhal number is to include the effects of frequency content in the data. This is expected to account for much of the unsteady effects seen in the measured data, and does a reasonable job, based on the model fits. Further improvement may be realized by analyzing the data retaining nonlinearities with roll rate and $\mathrm{k}$. Figure 22 shows an example of the rolling moment at constant $\mathrm{k}$ values for a range of roll rates. These data are obtained by oscillating the model at various amplitudes to develop a range of rates through the midpoint of the cycles at constant $\mathrm{k}$ values. The effect of $\mathrm{k}$, and nonlinearities associated with rate are seen in this data format. It is important to realize that data obtained for this format of analysis needs to be at constant $\mathrm{k}$ values. The result of simply varying oscillation frequency at a constant oscillation amplitude results in what could be very misleading data, as shown in figure 23 , if $\mathrm{k}$ effects are significant. Using nonlinear forced oscillation data, similar to that presented in figure 22, allows for more thorough comparisons with rotary data. Although data taken with the F-16XL and modern fighter configuration presented herein was insufficient for these comparisons, data obtained for another configuration included sufficient forced oscillation results to make comparisons. The results with this other configuration showed very similar trends with rotary balance data to that seen with the F-16XL oscillatory and ramp motion comparison presented earlier. At low k values, the trends of the forced oscillation derived data agreed well with the rotary balance data, and at high $\mathrm{k}$ values, large differences between the two data sets were apparent. It is important to note that, as before, these differences were primarily only important in the middle angle of attack range around stall.

Another important effect that has traditionally been neglected is the effect of sideslip on body axis damping or unsteady terms. Figure 24 shows an example of the effect of a non-zero mean angle of sideslip on the roll damping parameter (using the previously mentioned regression equation) as a function of $\mathrm{k}$ at $\alpha=30^{\circ}$. These data were obtained by oscillating the model with various combinations of sting roll angle offset and pitch angles. All data were obtained with oscillation amplitude of $30^{\circ}$. The data show that small sideslip values have no apparent effect on the coefficient, however at larger values of sideslip, large decreases in the damping parameter result, eventually even resulting in unstable roll rate damping. Additionally, at least through the moderate angles of sideslip, large variations of the damping parameter with $\mathrm{k}$ are seen - similar to the zero sideslip data shown previously.

These results highlight potential sources of errors in simulation modeling if the similitude requirements are not followed. For example, assume a flight time history motion of a periodic rolling maneuver at $\alpha=30^{\circ}$ and $30^{\circ}$ maximum roll angle, and $\mathrm{k}=0.1$. Using the standard linear superposition model previously discussed, and the data in figure 16, at the maximum roll angle, an error in rolling moment of over $38 \%$ (compared to measured wind tunnel data) is produced due to not including the effect of $\mathrm{k}$ on the "static" term, $\mathrm{C}_{\beta}$

6

American Institute of Aeronautics and Astronautics 
A limited evaluation of a simple model of roll damping - including frequency effects - was conducted as a first step in developing more comprehensive and robust modeling approaches. The roll damping parameter $\left(\mathrm{C}_{1}\right)$ and an increment to the conventional static lateral stability $\left(\Delta \mathrm{C}_{1}\right)$ was modeled as a function of angle of attack and reduced frequency based on the values shown in figure 25 . The reduced frequency was computed by curve fitting a sinusoidal response to the time history of roll rate at each time step in the simulation. Based on this approach, the frequency was computed as;

$$
\omega^{2} \cong \frac{-2\left(\dot{p}_{2}-\dot{p}_{1}\right)}{\left(p_{2}+p_{1}\right)\left(t_{2}-t_{1}\right)}
$$

and an average value of $\mathrm{k}$ for the previous one second was used for the table lookup. It was recognized that the calculation of frequency from dynamic aircraft motion time histories could provide misleading results and must be carefully interpreted. However, for the purposes of this study, this approach was satisfactory for initial evaluation of the aerodynamic effects discussed in this paper. Other studies are currently in progress that are focused on approaches for modeling time-dependent aerodynamic effects and implementation in simulation ${ }^{17,23}$.

Figure 26 shows time history outputs from a real-time simulation of a modern fighter airplane using the frequency-dependent roll damping model compared to a baseline model without the frequency-dependent effects. The computed values of $\mathrm{k}$ varied from 0 to 0.4 with a mean value of approximately 0.1 . The simulation results show differences in the motions as a result of using the frequency-dependent model indicating the importance of the frequency component on roll damping, even for relatively benign aircraft maneuvers. In addition, this relatively simple modeling approach produced smooth and realistic dynamic response. Future research is planned to evaluate the effects of this modeling approach on flight fidelity at high-angles-of-attack.

\section{Summary and Recommendations}

A consensus appears to be forming that the current wind tunnel testing and modeling process is not adequate for accurate prediction of flight dynamics of modern airplane designs operating at high angles of attack and with large angular rates. Many researchers are currently developing methods to try and improve on the current state-of-the-art. This paper has presented a few examples of recent results of large amplitude testing of fighter model configurations. The review of similitude requirements pointed out the need to maintain Strouhal number (reduced frequency) when comparing data sets. The effect of reduced frequency was configuration dependent, as well as functions of other state variables. Direct application of wind tunnel oscillatory data will require a method for determining an "equivalent" reduced frequency at any given point in time to allow the correct data to be looked up. An example of a simple representation of reduced frequency in simulation time history, and the application of a simple rolling moment model using this method was shown to be feasible. More complete models, including nonlinear effects of rate, and control surface deflections, and other state variables may require more sophisticated data table look-up approaches such as fuzzy logic or neural network schemes.

In order to develop better approaches to modeling the unsteady aerodynamic effects, flight validation of the models will be essential. This could effectively be accomplished using small, unpowered models that could be rapidly maneuvered in flight, and with sufficient data recording instrumentation to reduce the data. These same (or similar) models could then be tested using the wind tunnel test techniques available to generate the math models and compare directly with flight. Once this step is completed, other issues such as Mach number, Reynolds number and scale effects would need to be addressed. Another important area requiring more research, is the methodology of combining the various types of data - static, forced oscillation, rotary balance, and others which may be determined to be required - to approximate the actual, non-body axis maneuvers occurring in flight.

Conventional methods of combining forced oscillation and rotary balance data need to be addressed in light of the pronounced effects of reduced frequency the forced oscillation data may contain.

\section{References}

1. Ogburn, M.E.; and Foster, J.V.: Development of High-Angle-of-Attack Nose-Down Pitch Control Margin Design Guidelines for Combat Aircraft. NASA CP-10127, December 1993, pp. 293-322.

2. Cooperative Programme on Dynamic Wind Tunnel Experiments for Manoeuvering Aircraft. AGARDAR-305, October 1996.

3. Foster, J.V.: Investigation of the Susceptibility of Fighter Airplanes to the Out-of-Control Falling Leaf Mode. Presented at the NASA High Angle of Attack Conference, September 17-19, 1996.

4. Kalviste, Juri: Math Modeling of Aero Data for Aircraft dynamic Motion. AIAA 94-AFM-26-7, AIAA Atmospheric Flight Mechanics Conference, August 1994. 
5. Lorber, P.F.; and Carta, F.O.: Airfoil Dynamic Stall at Constant Pitch Rate and High Reynolds Number. AIAA 87-1329, June 1987

6. Galbraith, R.A.; Niven, A.J.; and Seto, L.Y.: On the Duration of Low Speed Dynamic Stall. ICAS86-2.4.3.

7. Francis, M.S. et al: An Investigation of Airfoil Dynamic Stall Overshoot of Static Airfoil Characteristics. AIAA-85-1773 August 1985.

8. Wolffelt, K.W.: Investigation on the Movement of Vortex Burst Position With Dynamically Changing Angle of Attack for a Schematic Deltawing in a Watertunnel With Correlation to Similar Studies in a Wind Tunnel. AGARD CP-413, October 1986.

9. Lambourne, N.C. et al: The Behavior of the Leading-Edge Vortices Over a Delta Wing Following a Sudden Change of Incidence. ARC$\mathrm{R} / \mathrm{M}-36451970$.

10. Gad-el-Hak, M.; and Ho, C.: The Pitching Delta Wing. AIAA Journal Vol. 23, No. 11, November 1985.

11. Reynolds, G.A.; and Abtahi, A.A.: Instabilities in Leading-Edge Vortex Development. AIAA-872424, August 1987.

12. Brandon, J.M.; and Shah, G.H.: Effect of Large Amplitude Pitching Motions on the Unsteady Aerodynamic Characteristics of Flat-Plate Wings. AIAA-88-4331, August 1988.

13. David, M.: Water Tunnel Investigation of the Vortex Dynamics of Periodically Pitched Wings. MS Thesis, Air Force Inst. Technology., December 1988. (Available from DTIC as AD-A206 359).

14. Brandon, J.M.; and Shah, G.H.: Unsteady Aerodynamic Characteristics of a Fighter Model Undergoing Large-Amplitude Pitching Motions at High Angles of Attack. AIAA-90-0309, January, 1990.

15. Cunningham, A.M.; and den Boer, R.G.: Harmonic Analysis of Force and Pressure Data Results For An Oscillating Straked Wing at High Angles. AIAA-87-2494, August, 1987.

16. Cunningham, A.M.; and Bushlow, T.: Steady And Unsteady Force Testing Of Fighter Aircraft Models In A Water Tunnel. AIAA-90-2815, August, 1990.

17. Klein, V., Murphy, P.C., Curry, T.J., and Brandon, J.M.: Analyis of Wind Tunnel Longitudinal Static and Oscillatory Data of the F-16XL Aircraft. NASA TM-97-206276, December 1997.

18. Myatt, J.H.: Modeling the Rolling 65-Degree Delta Wing with Critical State Encounters. AIAA 97-3646, August 1997.

19. McKeehan, P.D., Myatt, J.H., Grismer, D.S., and Buffington, J.M.: Simulation of a Tailless Aircraft Using Nonlinear Indicial Response Aerodynamic Modeling Methods. AIAA 97-3790, August 1997.
20. Hu, C., Lan, C.E., and Brandon, J.M.: Unsteady Aerodynamic Models for Maneuvering Aircraft. AIAA 93-3626, Monterey, CA August 9-11, 1993.

21. Lin Guo-Feng, Lan C. E., and Brandon, J.M.: A Generalized Dynamic Aerodynamic Coefficient Model for Flight Dynamics Applications. AIAA 97-3643, August, 1997.

22. Wang, Z.; Lan, C.E.; and Brandon, J.M.: Fuzzy Logic Modeling of Nonlinear Unsteady Aerodynamics. AIAA 98-4351, August, 1998

23. Fan, Y.; and Lutz, F.H.: Identification of an Unsteady Aerodynamic Model at High Angles of Attack. AIAA 96-3407, July, 1996.

24. Klein, V., Batterson, J.G., and Murphy, P.C.: Determination of Airplane Model Structure From Flight Data by Using Modified Stepwise Regression. NASA TP 1916, October 1981.

25. Campbell, J.P., Johnson, J.L., Jr., and Hewes, D.E.: Low-Speed Study of the Effect of Frequency on the Stability Derivatives of Wings Oscillation in Yaw With Particular Reference to High Angle-ofAttack Conditions. NACA RM L55H05, November 1955.

26. Grafton, S.B.: Wind-Tunnel Free-Flight Investigation of a Model of a Cranked-ArrowWing Fighter Configuration. NASA TP-2410, 1985 .

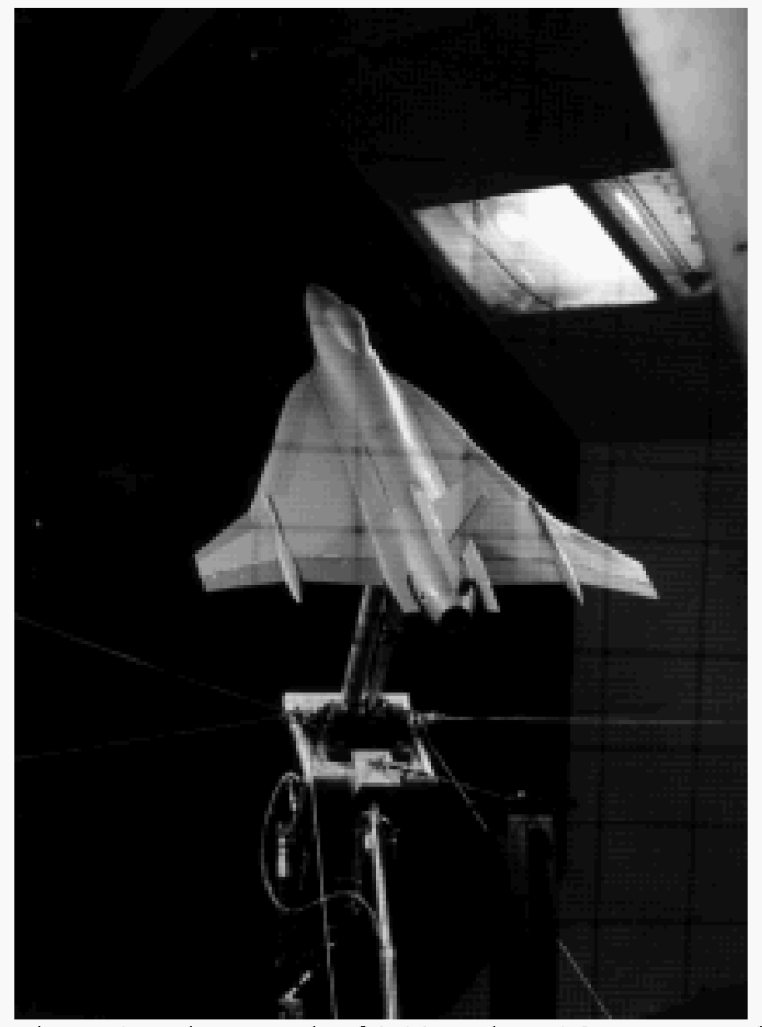

Figure 1 - Photograph of 0.10-scale F-16XL mounted for large amplitude pitching tests. 


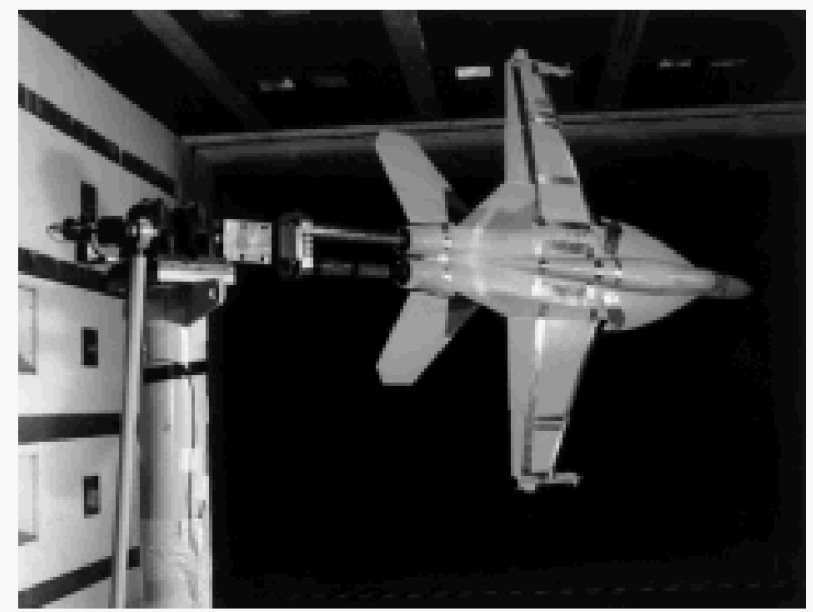

Figure 2 - Figure of modern fighter model mounted for roll oscillations in the $14 \times 22$ Subsonic Tunnel.

\begin{tabular}{|c|c|c|c|}
\hline $\mathrm{C}_{\mathrm{i}}=\mathrm{f}($ & $\begin{array}{l}\alpha, \beta \\
\delta \\
V / V_{\text {sound }} \\
\rho V 1 / \mu\end{array}$ & $\begin{array}{l}\text { aircraft attitude relative to free stream } \\
\text { control deflection } \\
\text { Mach number } \\
\text { Reynolds number }\end{array}$ & $\begin{array}{l}\text { Static } \\
\text { model }\end{array}$ \\
\hline & $\Omega 1 / V$ & reduced angular velocity & \multirow{2}{*}{$\begin{array}{l}\text { Rotary balance } \\
\text { Forced oscillation }\end{array}$} \\
\hline & $\omega \mathrm{l} / \mathrm{V}$ & reduced osc. freq., $\mathrm{k}$ (Strouhal number & \\
\hline & $\begin{array}{l}\mathrm{al} / \mathrm{N}^{2} \\
\Omega_{\text {dol }} \mathrm{l}^{2} / \mathrm{V}^{2} \\
\mathrm{~V}^{2} / \mathrm{g} \\
\mathrm{m} / \rho \mathrm{l}^{3} \\
\mathrm{I} / \mathrm{\rho l}^{5} \\
\end{array}$ & $\begin{array}{l}\text { reduced linear acceleration } \\
\text { reduced angular acceleration } \\
\text { Froude number } \\
\text { Relative density factor } \\
\text { Relative mass moment of inertia }\end{array}$ & \multirow[t]{3}{*}{$\begin{array}{l}\text { Dynamic } \\
\text { Model }\end{array}$} \\
\hline & $E I^{\prime} / \rho V^{2} 1^{4}$ & Aeroelastic bending parameter & \\
\hline & $\begin{array}{l}\mathrm{GJ} / \rho \mathrm{V}^{2} 1^{4} \\
\mathrm{tV} / 1\end{array}$ & $\begin{array}{l}\text { Aeroelastic torsion parameter } \\
\text { Reduced-time parameter ) }\end{array}$ & \\
\hline
\end{tabular}

Figure 3 - Similitude requirements
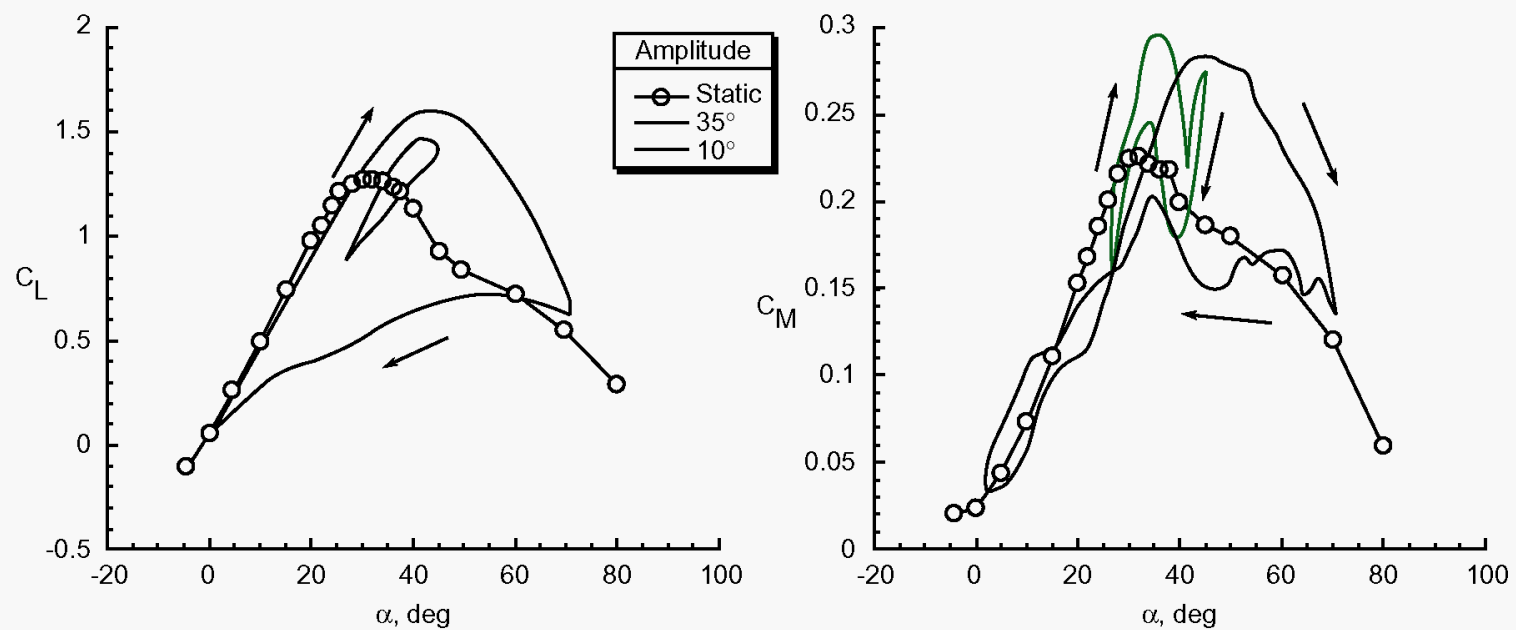

Figure 4 - Pitch oscillation results. F-16XL, $\hat{\mathrm{q}}_{\max }=0.02, \alpha_{0}=36^{\circ}$

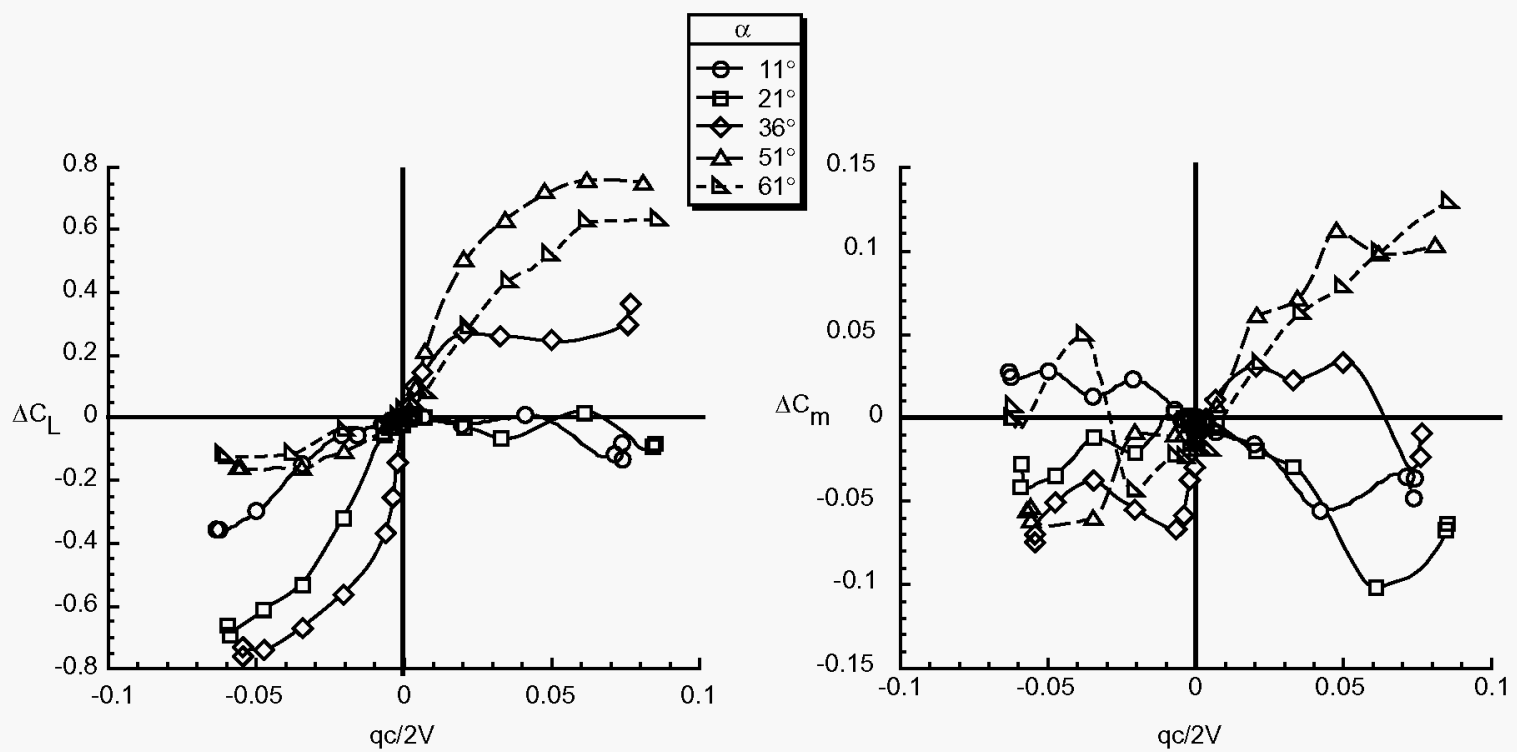

Figure 5 - Effect of pitch rate from ramp motions. F-16XL. 

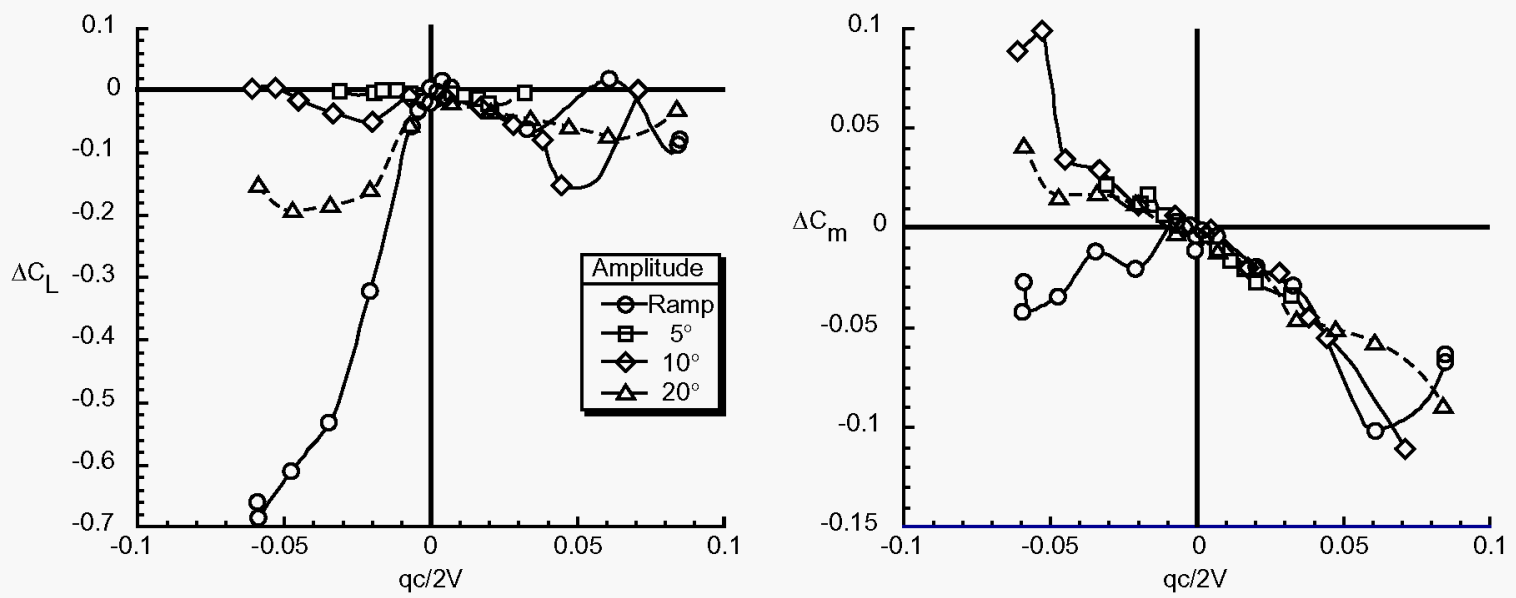

Figure 6 - Oscillation amplitude effects. F-16XL, $\alpha_{0}=21^{\circ}$.
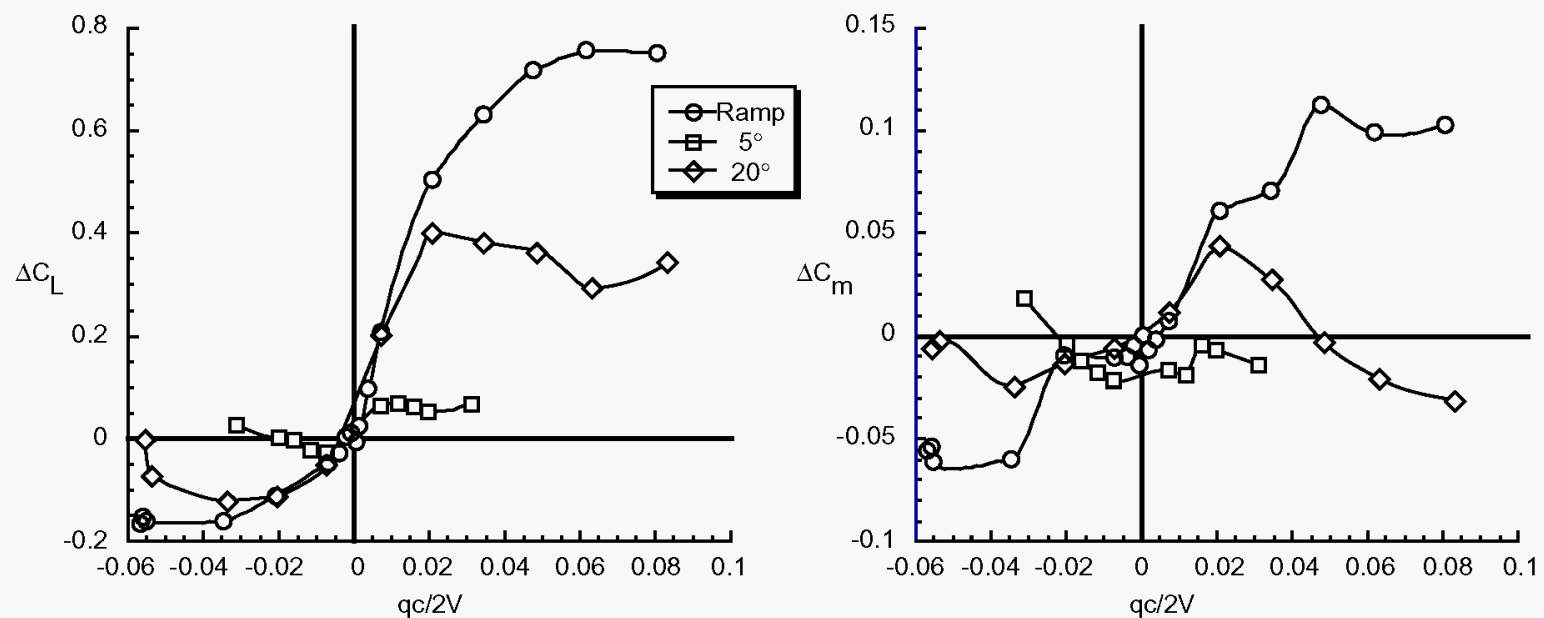

Figure 7 - Oscillation amplitude effects. F-16XL, $\alpha_{0}=51^{\circ}$.

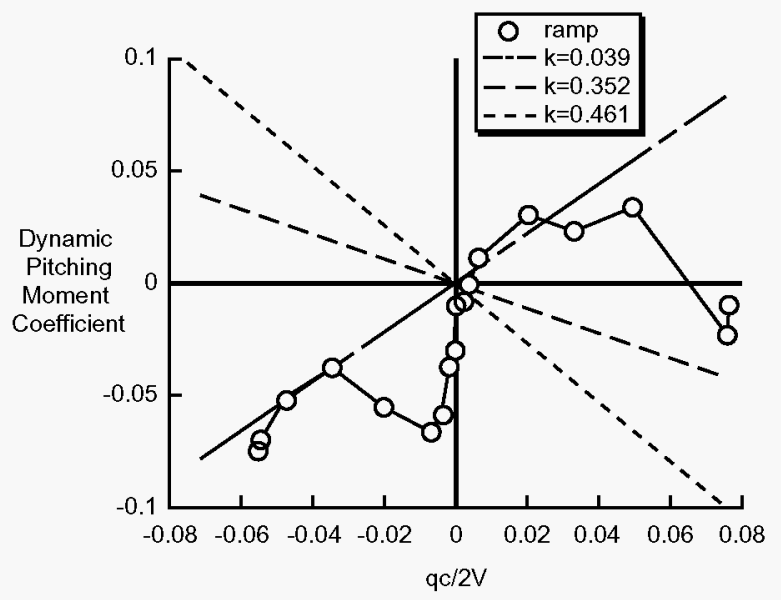

Figure 8 - Comparison of ramp and linearized damping parameter. $\alpha_{0}=36^{\circ}$, F-16XL 

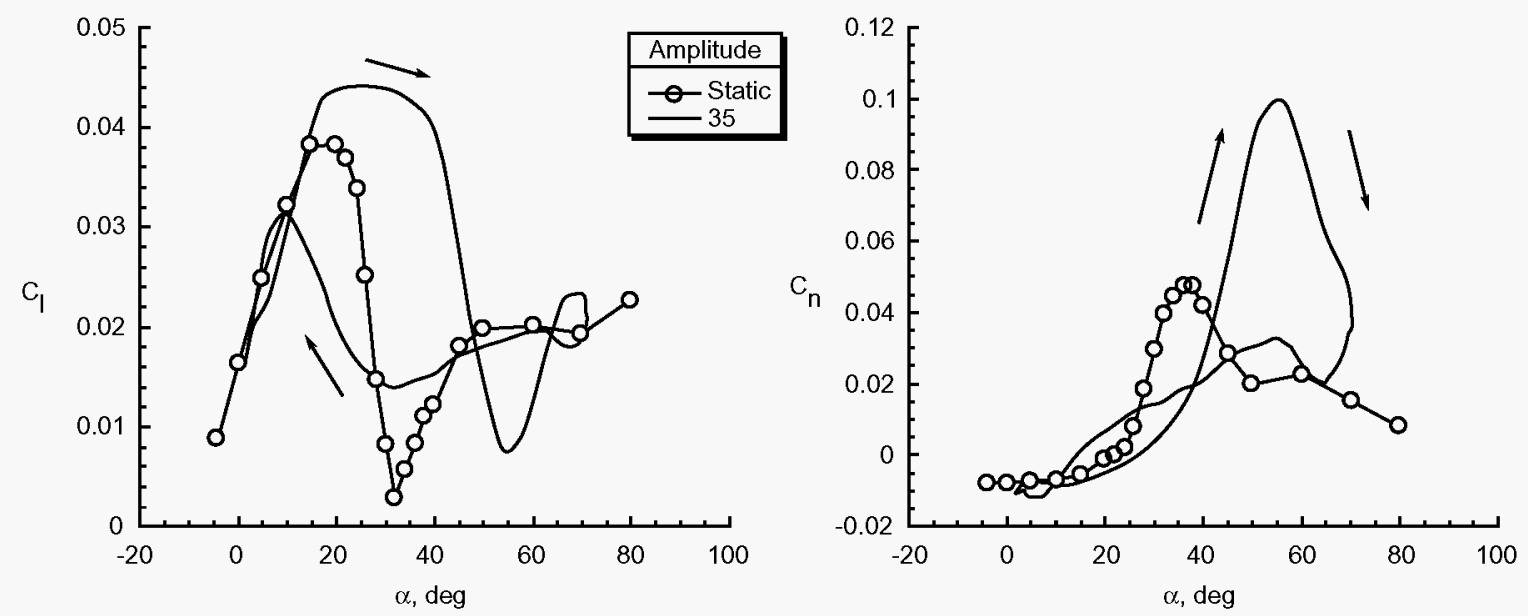

Figure 9 - Lateral-directional coefficients during pitch oscillation at $\beta=-10^{\circ}$, F-16XL.
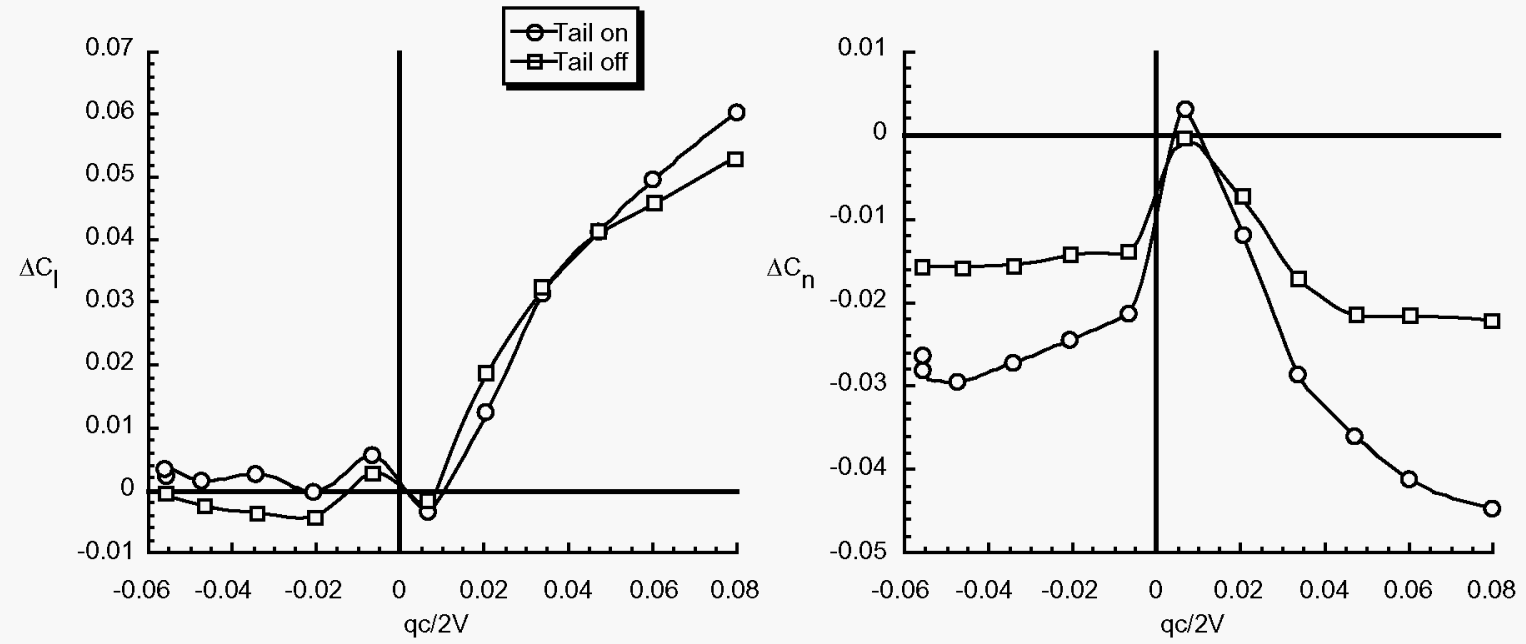

Figure 10 - Effect of the vertical tail during pitch oscillations at $\beta=-20^{\circ}, \mathrm{F}-16 \mathrm{XL}$.

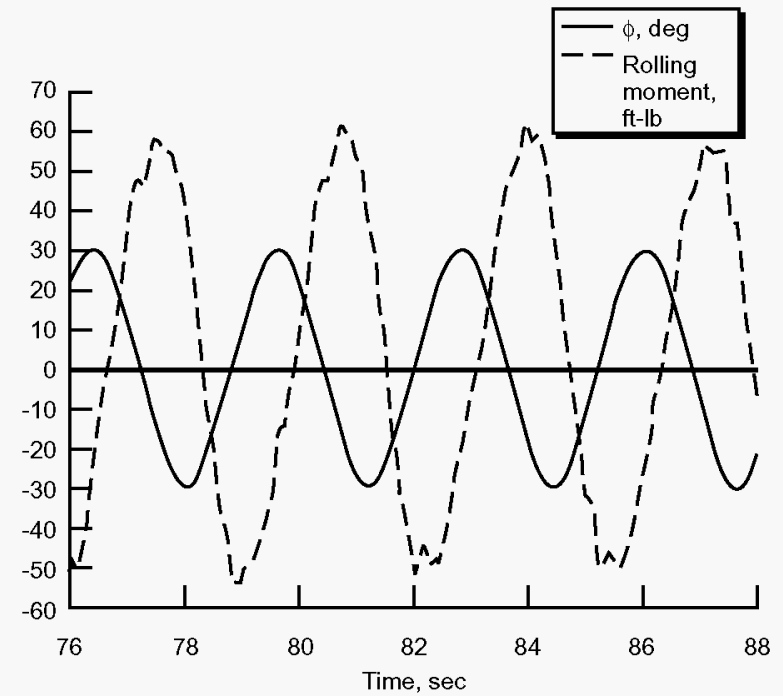

Figure 11 - Time history of rolling moment during roll oscillation test. F-16XL, $\alpha=34^{\circ}$.

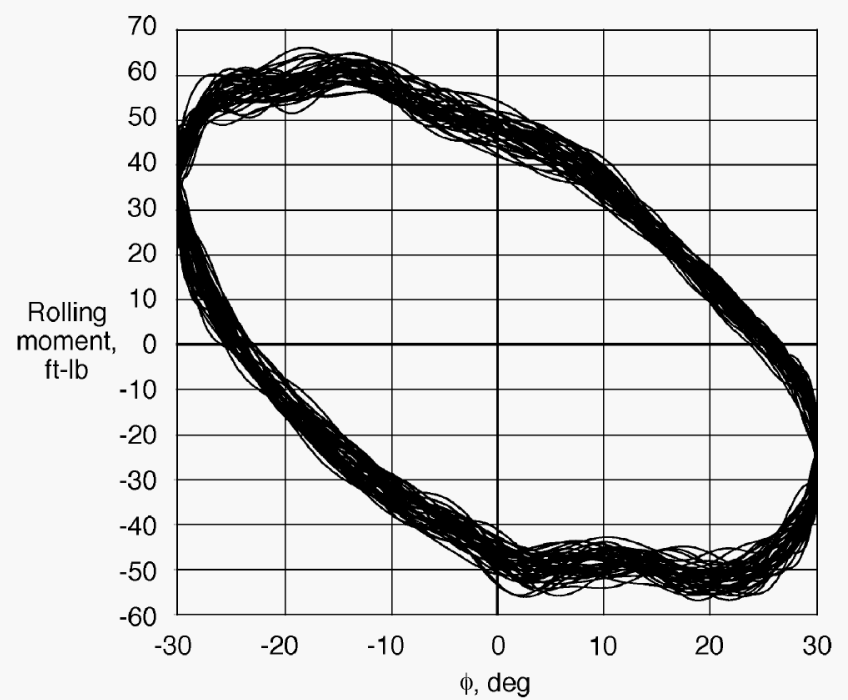

Figure 12 - Rolling moment coefficient response with roll angle. F-16XL, $\alpha=34^{\circ}, \hat{\mathrm{p}}_{\max }=0.04$. 


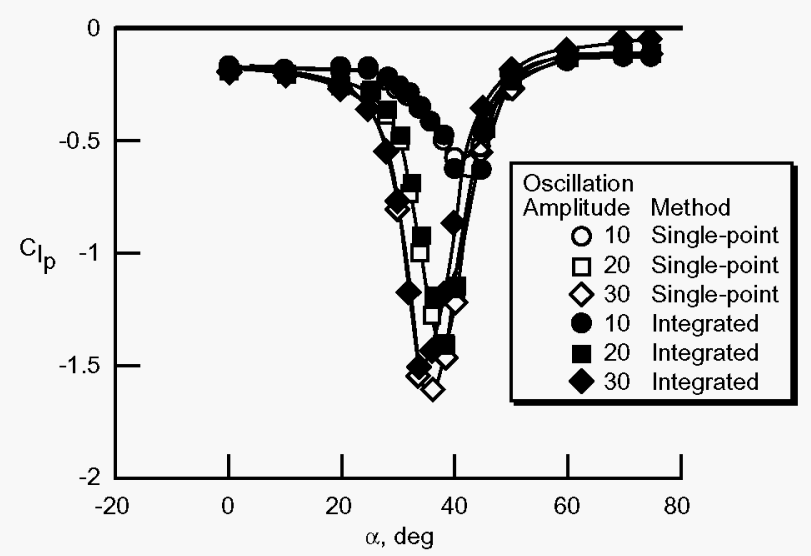

Figure 13 - Comparison of integrated and single point methods of computing roll damping parameter. F$16 \mathrm{XL}$.
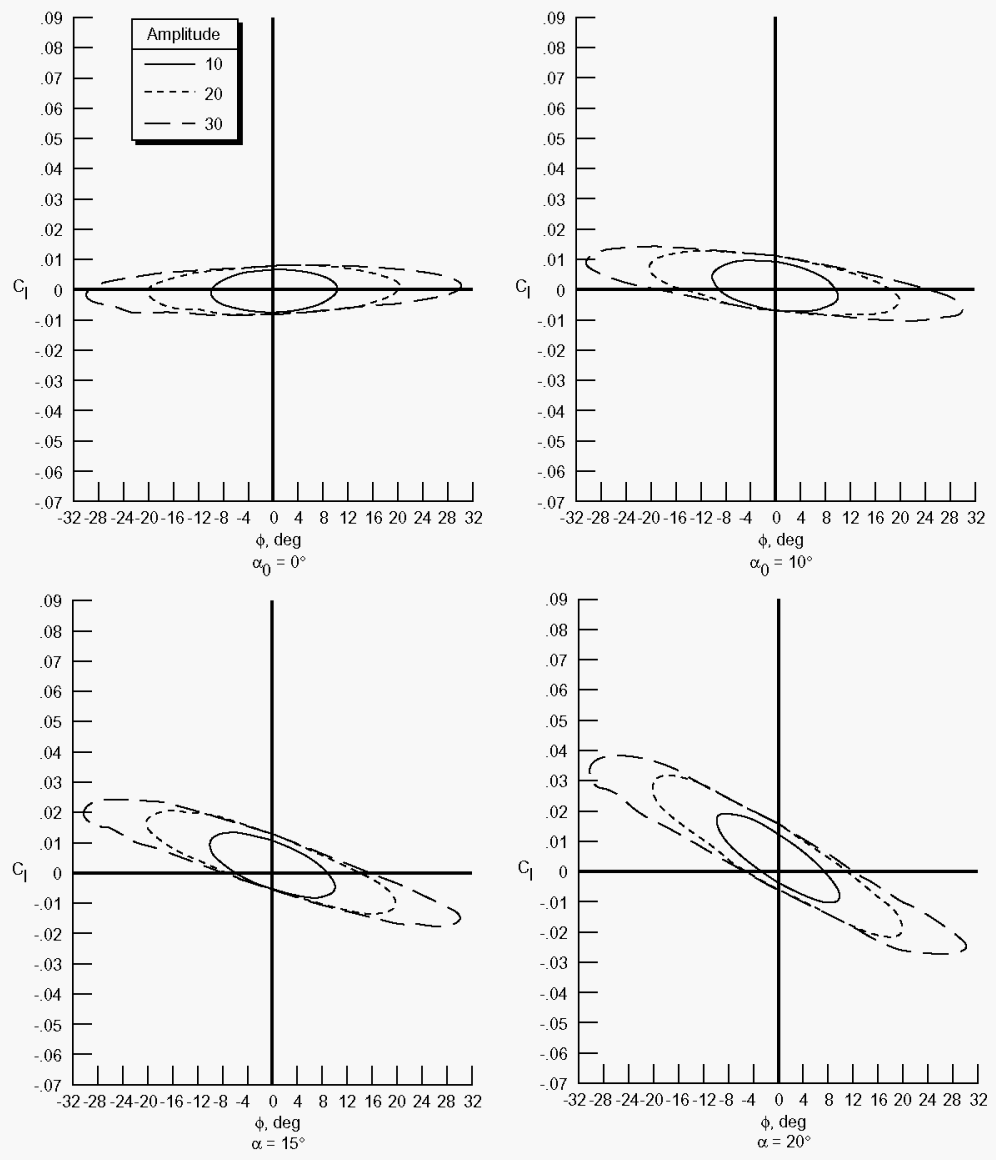

a. Low angle of attack

Figure $14-$ Rolling moment response. F-16XL, $\hat{\mathrm{p}}_{\max }=0.04$. 

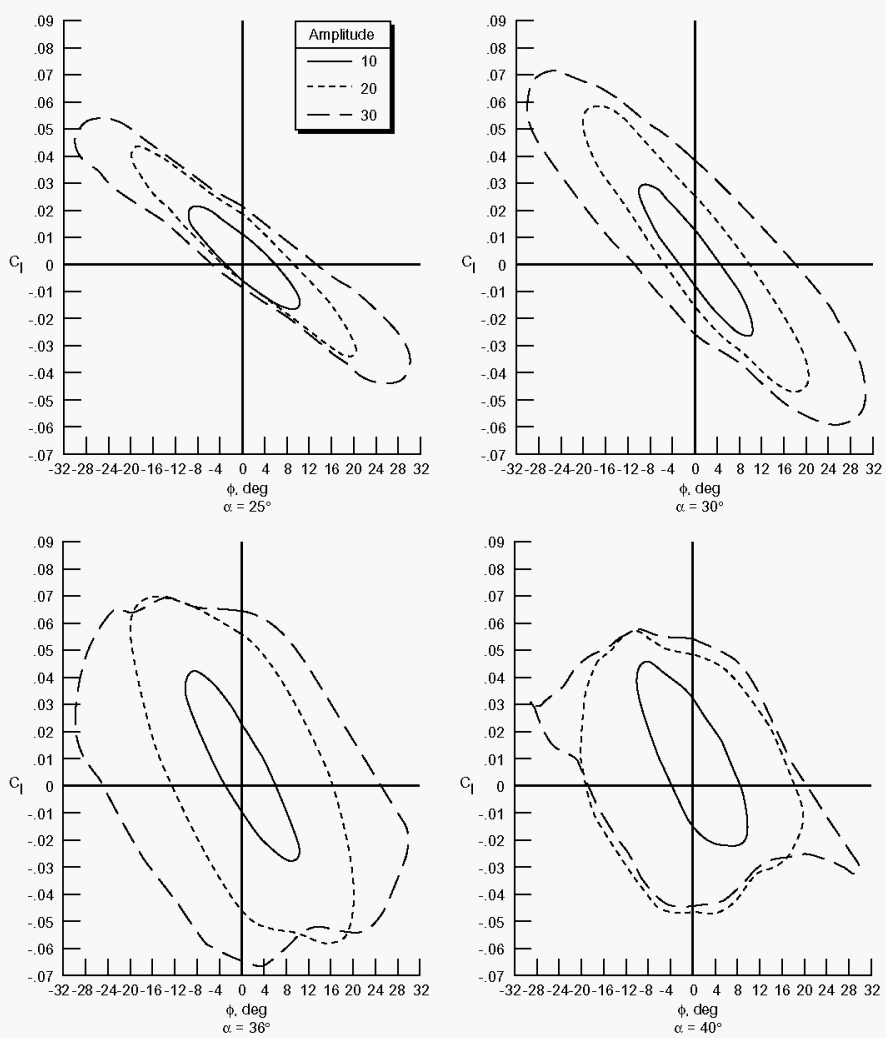

b. Moderate angle of attack

Figure 14 - continued.
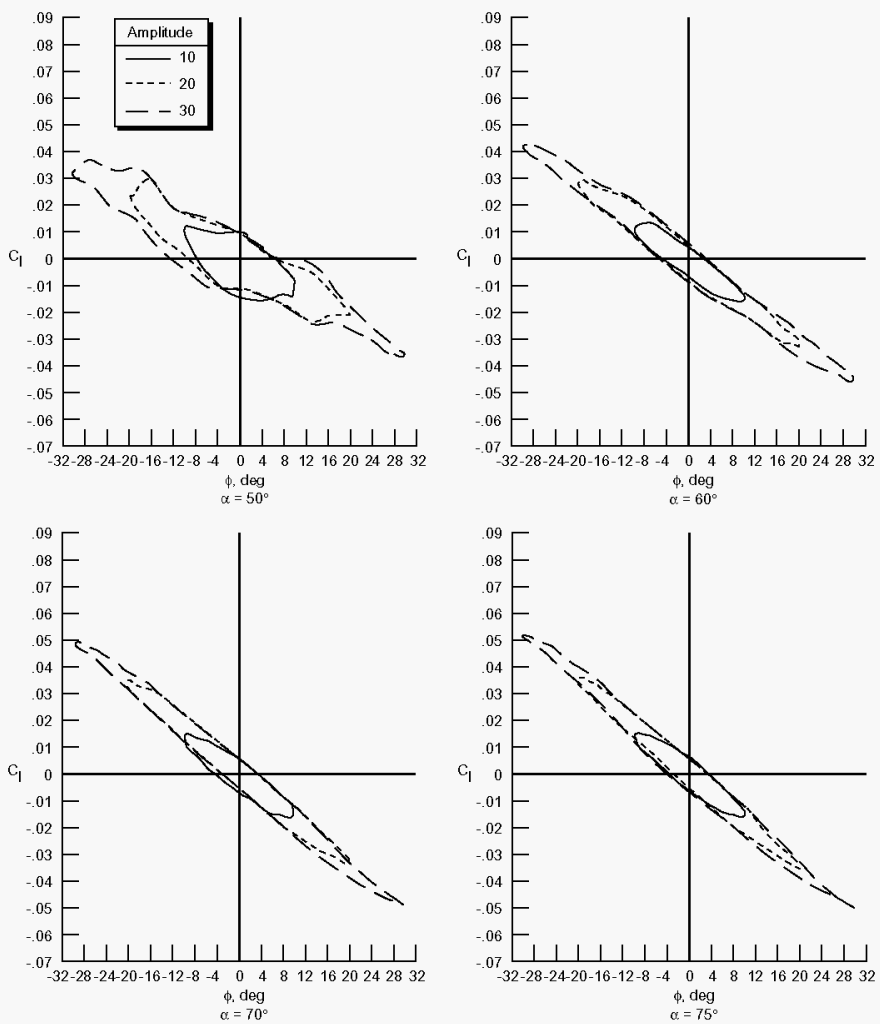

c. High angle of attack

Figure 14 - concluded. 

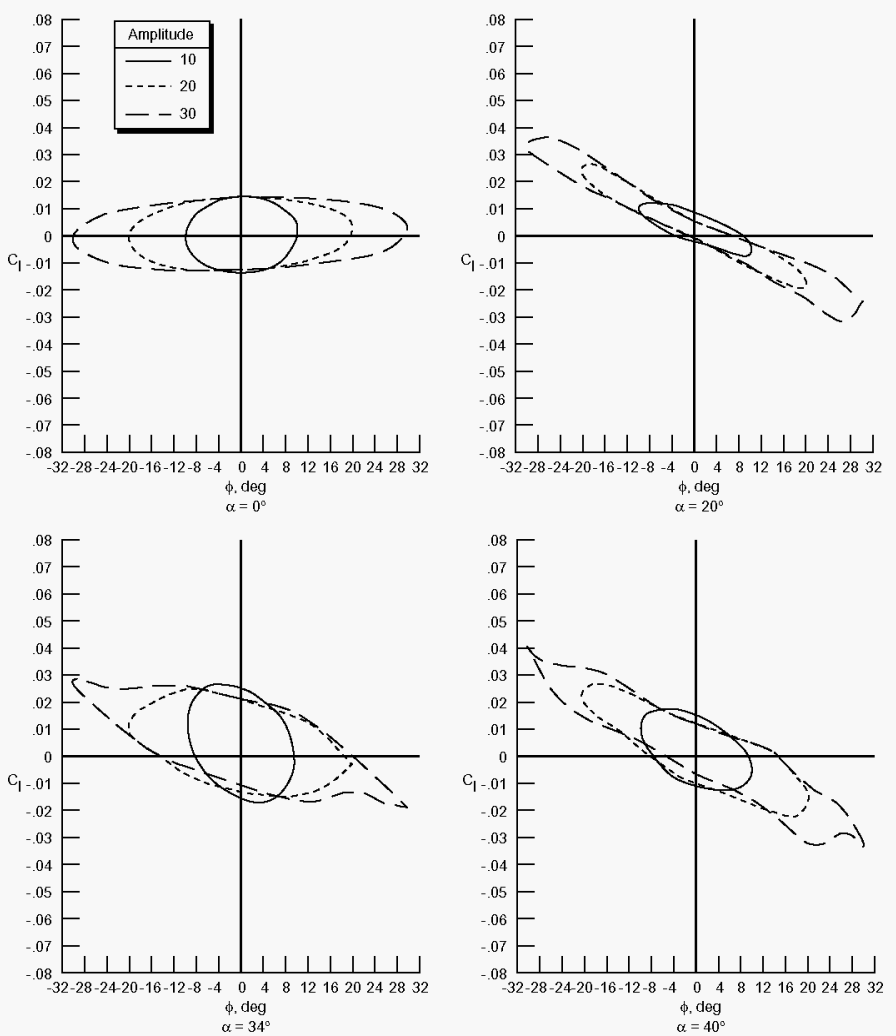

a. Low and moderate angle of attack

Figure 15 - Rolling moment response. Modern fighter configuration model, $\hat{\mathrm{p}} \max =0.04$.
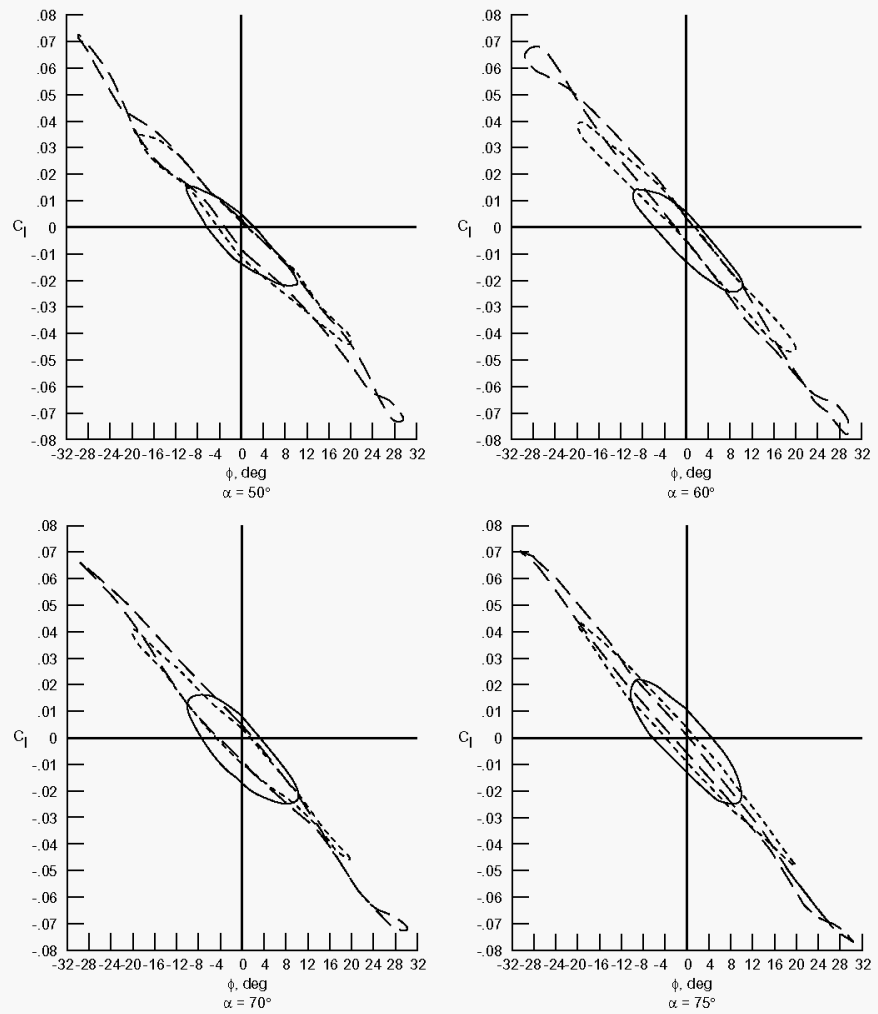

b. High angle of attack

Figure 15 - concluded. 
AIAA - 98-4447

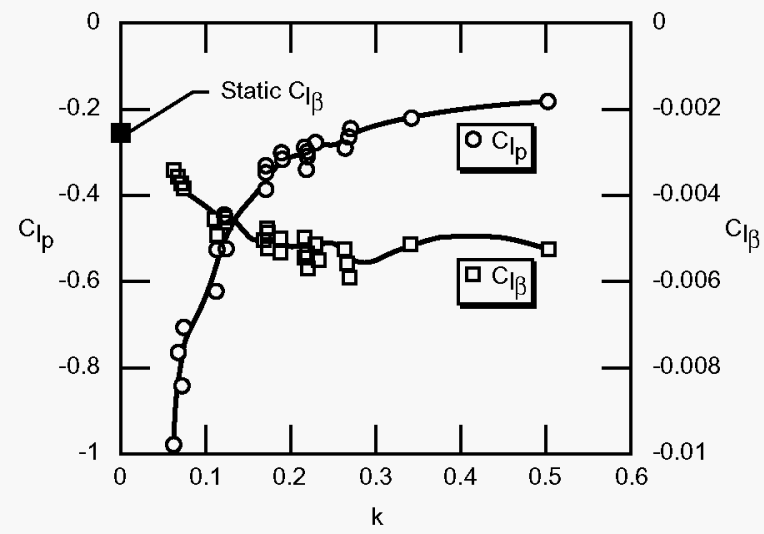

Figure 16 - Effect of reduced frequency on rolling moment derivatives. F-16XL, $\alpha=30^{\circ}$.

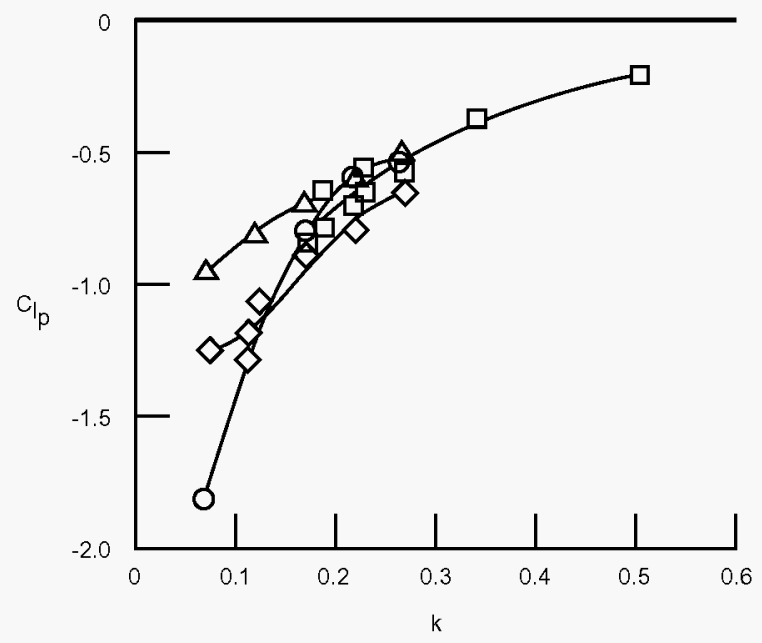

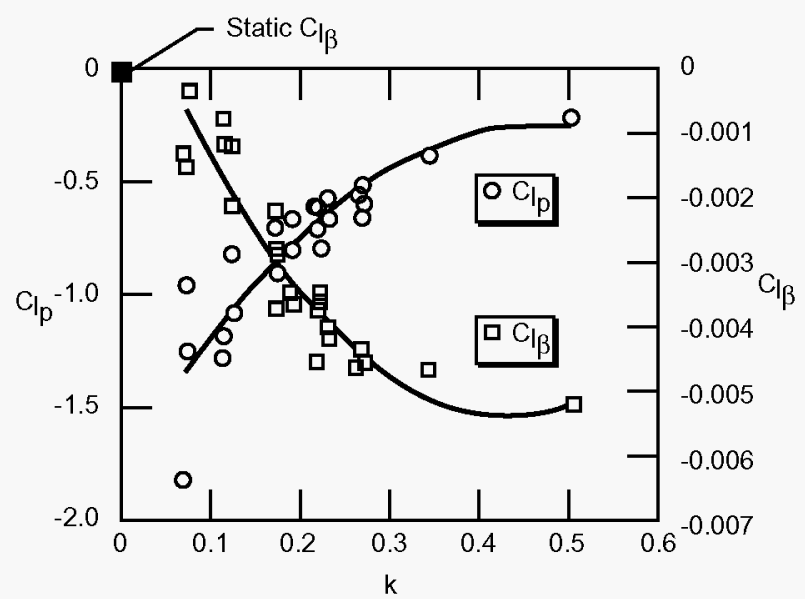

Figure 17 - Effect of reduced frequency on rolling moment derivatives. F-16XL, $\alpha=40^{\circ}$.

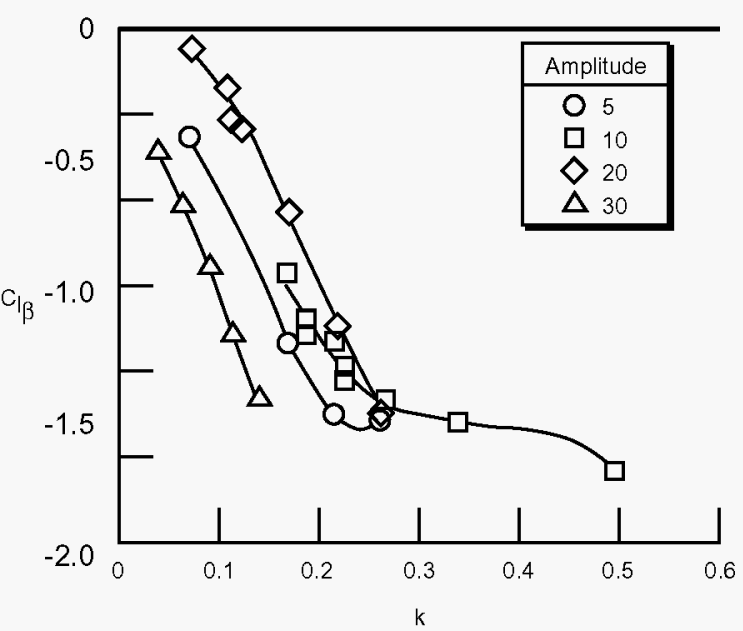

Figure 18 - Amplitude effects on rolling moment derivatives during roll oscillations. F-16XL, $\alpha=40^{\circ}$.

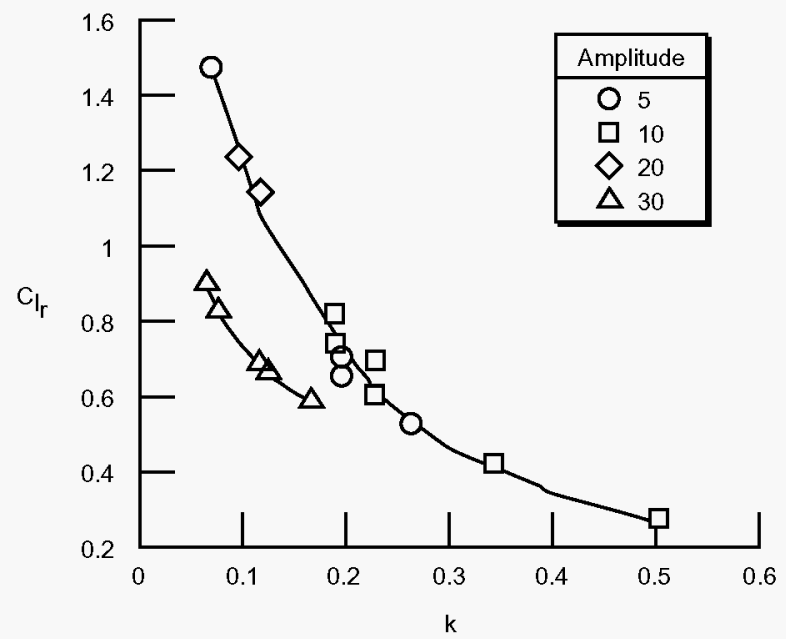

Figure 19 - Amplitude effects on rolling moment derivative during yaw oscillations. F-16XL, $\alpha=40^{\circ}$. 

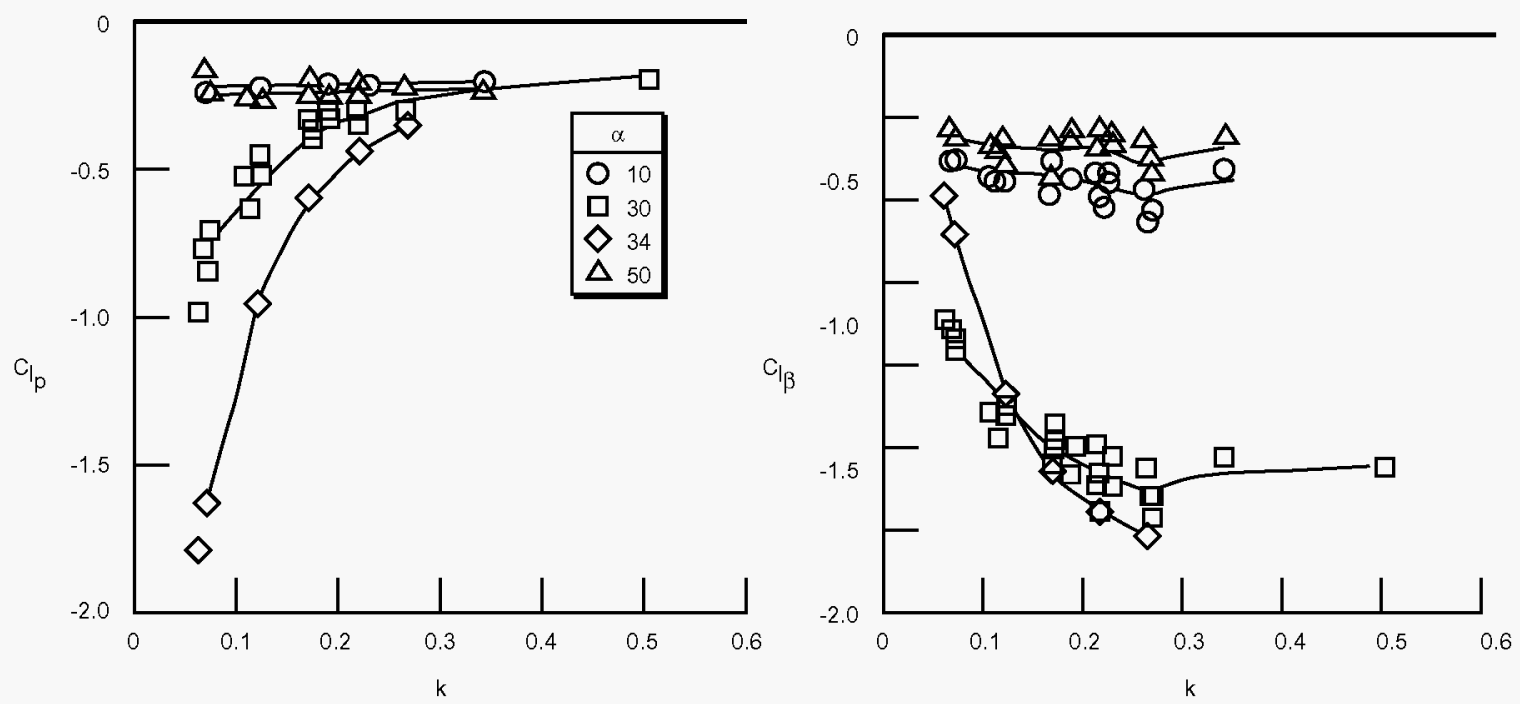

Figure 20 - Effect of angle of attack and reduced frequency on rolling moment derivatives. F-16XL.

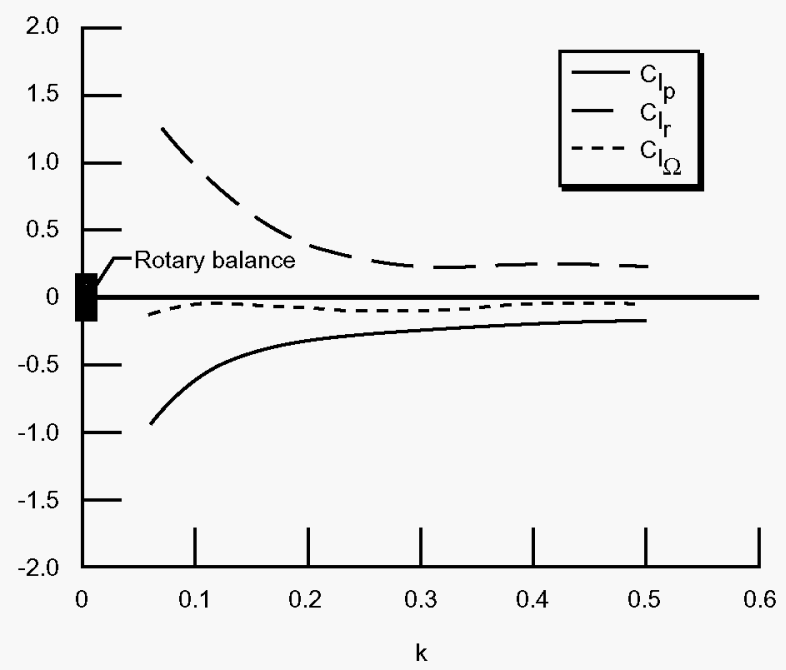

Figure 21 - Comparison of forced oscillation and rotary balance results. F-16XL, $\alpha=30$.

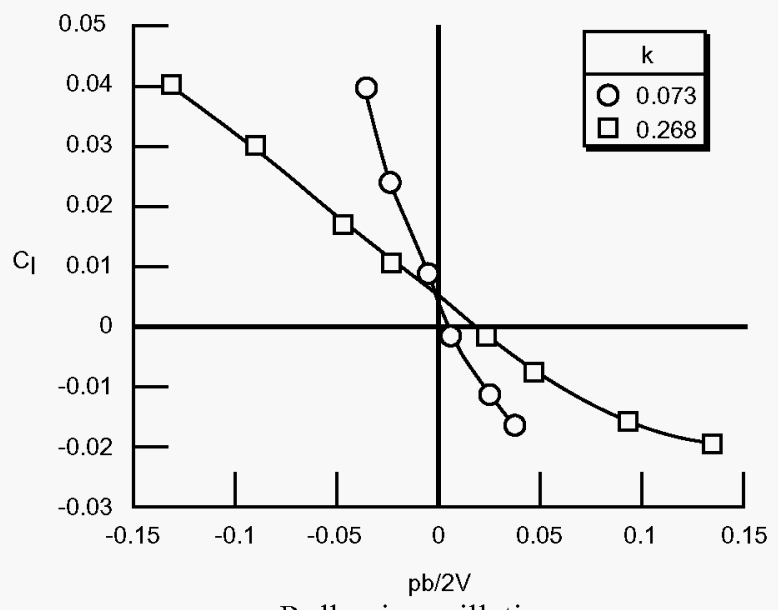

a. Roll axis oscillation

Figure 22 - Non-linear rolling moment results. F-16XL

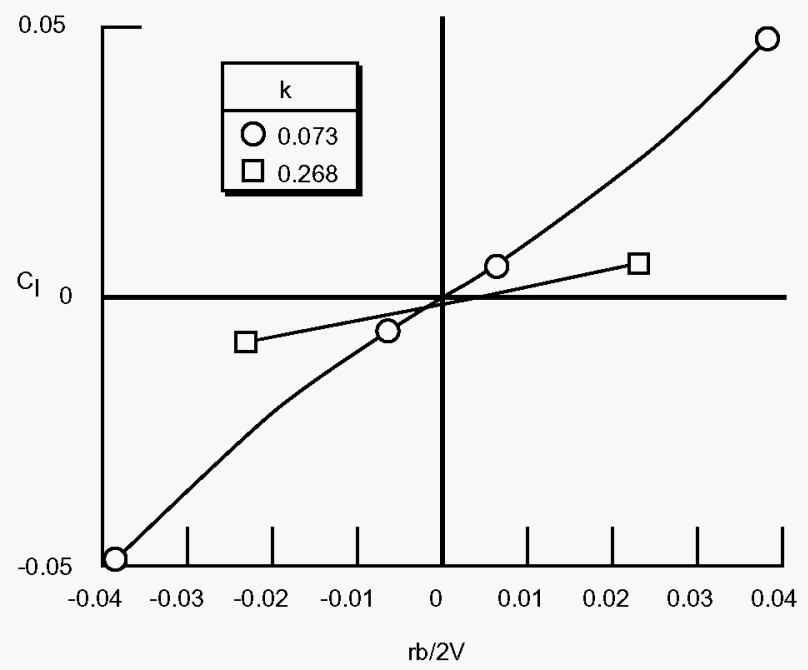

b. Yaw axis oscillation

Figure 22 - concluded.

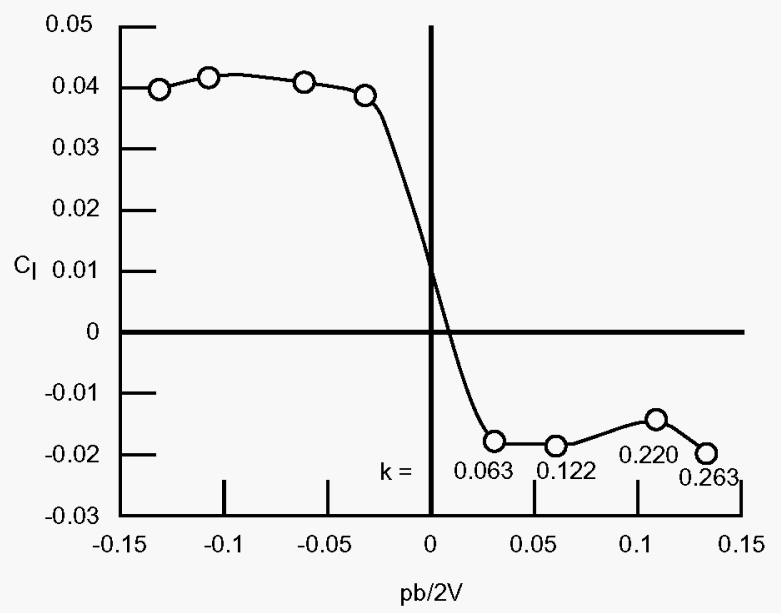

Figure 23 - Rolling moment results using constant amplitude oscillations. F-16XL. 


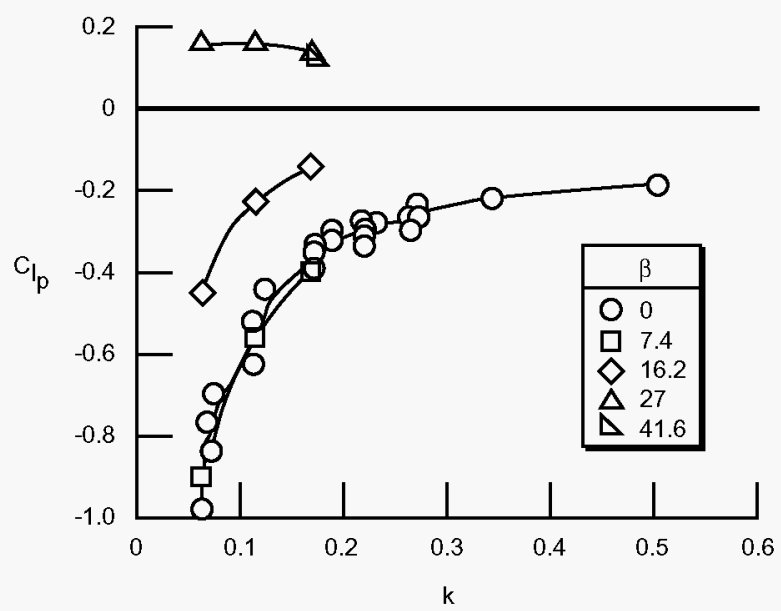

Figure 24 - Effect of sideslip on roll damping derivative. F-16XL, $\alpha=30^{\circ}$.
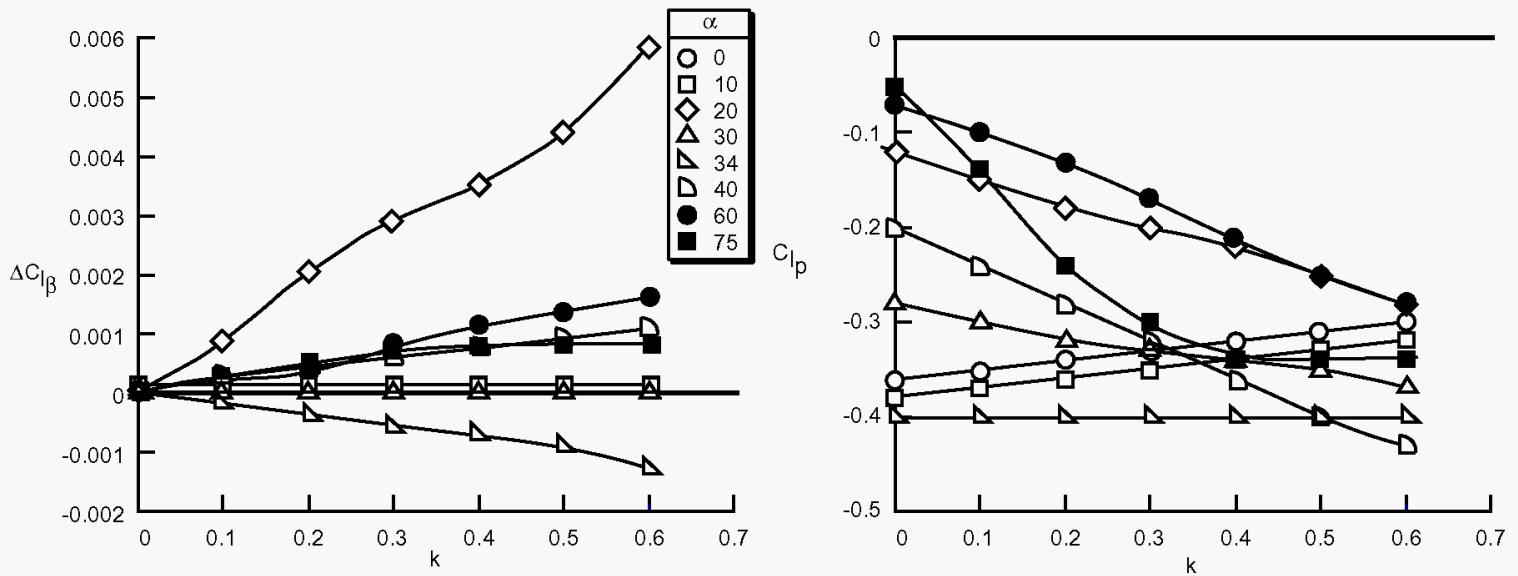

Figure 25 - Roll damping model used for simulation. Modern fighter configuration model.
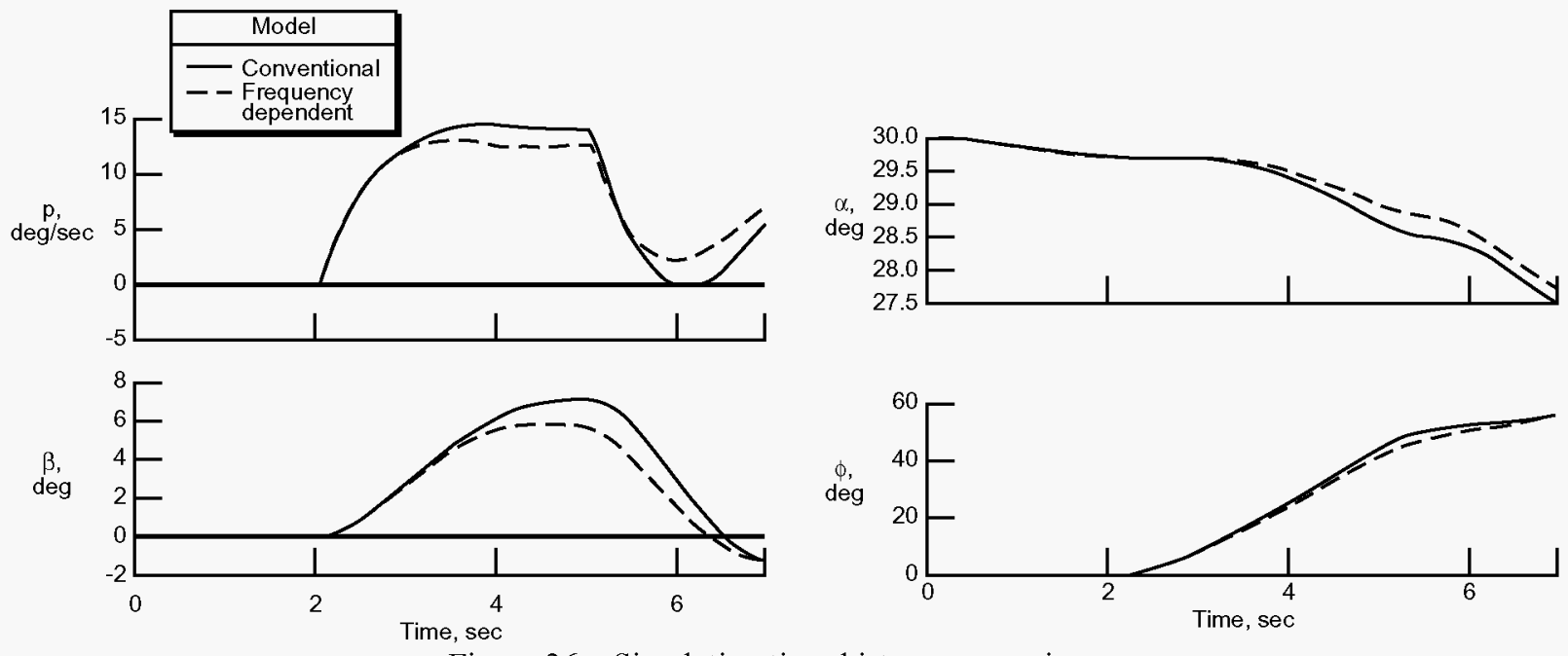

Figure 26 - Simulation time-history comparisons. 